\title{
Contracaecum larvae: morphological and morphometric retrospective analysis, biogeography and zoonotic risk in the amazon
}

Larva de Contracaecum: análise retrospectiva morfológica e morfométrica, biogeografia e risco zoonótico na amazônia

\author{
Raul Henrique da Silva Pinheiro ${ }^{1,2}$; Adriano Penha Furtado ${ }^{1,3}$; Jeannie Nascimento dos Santos ${ }^{1,3}$; \\ Elane Guerreiro Giese ${ }^{1,2 *}$
}

\author{
${ }^{1}$ Programa de Pós-Graduação em Biologia de Agentes Infecciosos e Parasitários, Instituto de Ciências Biológicas, Universidade Federal \\ do Pará - UFPA, Belém, PA, Brasil \\ ${ }^{2}$ Laboratório de Biologia Celular e Helmintologia "Profa Dra Reinalda Marisa Lanfredi”, Instituto de Ciências Biológicas, \\ Universidade Federal do Pará - UFPA, Belém, PA, Brasil \\ ${ }^{3}$ Laboratório de Histologia e Embriologia Animal, Instituto da Saúde e Produção Animal, Universidade Federal Rural da Amazônia - \\ UFRA, Belém, PA, Brasil
}

Received October 22, 2018

Accepted December 18, 2018

\begin{abstract}
In Brazil there are several records of parasitic nematodes of fish with zoonotic potential, especially those belonging to the family Anisakidae. This study considers the morphology, morphometry and prevalence of Contracaecum in Astronotus ocellatus, fish consumed in the Amazon and sold as ornamental and it also performs a retrospective analysis of the diversity of fish with larvae of Contracaecum, in studies carried out in Brazil over a period of 90 years. 40 specimens of $A$. ocellatus were necropsied, and the nematodes were collected and fixed in 93 parts $70 \%$ ethyl alcohol, 5 parts formaldehyde, and 2 parts glacial acetic acid (AFA) for morphological analysis under light microscopy and scanning electron microscopy. Of the 40 fish collected during this work, 27 were parasitized by Contracaecum larvae with a total intensity of 150 larvae. Retrospective analysis of intermediate host diversity for Contracaecum larvae resulted in 16 orders, 49 families, 96 genera, 140 species and a hybrid morphotype. In the retrospective study, half of the fish were from freshwater, with the order Perciformes being the most representative, with 16 families, 30 genera and 37 species. In Brazil, the occurrence of larvae of Contracaecum in fish was reported in 15 of the 26 states, with Rio de Janeiro presenting the most information regarding fish harboring Contracaecum larvae.
\end{abstract}

Keywords: Astronotus ocellatus, fish hygiene, zoonosis, Pará.

\section{Resumo}

No Brasil existem vários registros de nematóides parasitos de peixes com potencial zoonótico, especialmente aqueles pertencentes à família Anisakidae. Este estudo considera a morfologia, morfometria e prevalência de Contracaecum em Astronotus ocellatus, peixe consumido na Amazônia e vendido como ornamental, e também realiza uma análise retrospectiva da ictiofauna parasitada com larvas de Contracaecum, em estudos realizados no Brasil, durante um período de 90 anos. 40 espécimes de $A$. ocellatus foram necropsiados, e os nematóides foram coletados e fixados em 93 partes de álcool etílico 70\%, 5 partes de formaldeído e 2 partes de ácido acético glacial (AFA) para análise morfológica em microscopia de luz e microscopia eletrônica de varredura. Dos 40 peixes coletados durante este trabalho, 27 estavam parasitados por Contracaecum, com intensidade total de 150 larvas. Análises retrospectivas da diversidade de hospedeiros intermediários para larvas de Contracaecum resultaram em 16 ordens, 49 famílias, 96 gêneros, 140 espécies e um morfotipo híbrido.

\footnotetext{
*Corresponding author: Elane Guerreiro Giese. Laboratório de Histologia e Embriologia Animal, Instituto da Saúde e Produção Animal, Universidade Federal Rural da Amazônia - UFRA, Avenida Presidente Tancredo Neves, 2501, Terra Firme, CEP 66077-830, Belém, PA, Brasil. e-mail: elane.giese@ufra.edu.br
} 
No estudo retrospectivo, metade dos peixes eram de água doce, sendo a ordem Perciformes a mais representativa, com 16 famílias, 30 gêneros e 37 espécies. No Brasil, a ocorrência de larvas de Contracaecum em peixes foi relatada em 15 dos 26 estados, com o Rio de Janeiro apresentando mais informaçóes sobre peixes portadores de larvas de Contracaecum.

Palavras-chaves: Astronotus ocellatus, higiene do pescado, zoonose, Pará.

\section{Introduction}

Astronotus ocellatus (Agassiz, 1831) (Cichliformes: Cichlidae) is a species native to the Amazon basin. Its culture was stimulated in the 1930s by governmental policies aimed at establishing this species in the South and Southeast river basins of the country (FONTENELE \& NEPOMUCENO, 1983), due its their economic value (AZEVEDO et al., 2007) and trophic plasticity (FIROUZBAKHSH et al., 2011).

Fish consumption is a good option for healthy eating, but consumption of raw or cold-smoked fish combined with deficient sanitary quality may make fish consumption a public health problem (KNOFF et al., 2013), since Amazonian fish have a large parasitic fauna. In Brazil, there are several records of the Anisakidae and Raphidascarididae families in marine fish, with parasites possessing zoonotic potential (FONTENELLE et al., 2013). Considering the great representativeness of the Anisakidae family in commercially important fish in Brazil, the Ministério da Saúde in 2010 classified the biological risk of Anisakidae infection as belonging to Risk Class 2, since they are parasites with moderate risk and limited risk of transmission (BRASIL, 2010). Infections by Anisakidae larvae result in a combination of two factors: direct action of larvae during tissue invasion and interactions between the host's immune system and the substances released by the parasite or the host's immune response to its presence (UBEIRA et al., 2000).

Although there are many studies related to larvae of the family Anisakidae in marine fish, the occurrence, data on the prevalence and morphology of larvae of this family in freshwater fish are still scarce, when compared to the numbers of freshwater environments and diversity of fish present in these habitats, distributed in the different states of Brazil. The objective of this study is to characterize the morphology, morphometry, biogeography and prevalence of nematoda Anisakidae in A. ocellatus, commercialized in the municipality of Santarém, Pará, in the Brazilian Amazon, in addition to gathering and analyzing retrospective data on the Brazilian ichthyofauna that hosts larvae of Contracaecum sp.

\section{Materials and Methods}

Forty $A$. ocellatus [total length 19-29 (23) cm; weight 175-514 (354) g] specimens were obtained. Fish were captured by artisanal fishermen in Tapajós river in the municipality of Santarém (2²6'22”S: 5441'55’W'), Pará State (Fig. 1). The fish were transported in an isothermal box to the Laboratório de Histologia e Embriologia Animal, Instituto de Saúde e Produção Animal, Universidade Federal do Rural da Amazônia, City of Belém for necropsy. After biometric analyses, the animals were necropsied for helminths. The digestive tract of each specimen was isolated in a Petri dish containing physiological solution and analyzed using stereomicroscope (LEICA-ES2). The nematode larvae found dead were fixed in AFA solution (93 parts 70\% ethyl alcohol, 5 parts formaldehyde, and 2 parts glacial acetic acid), stored at room temperature. For morphological and morphometric analysis ten larvae were dehydrated in an ethanol series, clarified with lactophenol, placed on a microscope slide under a coverslip as a temporary mount, observed using a light microscope, and photographed using a LEICA DM2500 microscope with an imaging capture system. Measurements are shown in micrometers as the mean followed by the range, or as otherwise indicated. Taxonomic classification of nematodes was in accordance with Moravec (1998), Timi et al. (2001), Felizardo et al. (2009), and Fonseca et al. (2016).

For scanning electron microscopy, six larvae were washed in phosphate-buffered saline ( $\mathrm{pH} 7.0$ ), post-fixed in $1 \%$ osmium tetroxide, dehydrated to the critical point of $\mathrm{CO}_{2}$, metalized with gold-palladium, and analyzed with the VEGA 3 LMU/TESCAN scanning electron microscope at the Laboratório de Microscópia Eletrônica de Varredura, Instituto da Saúde e Produção Animal - Universidade Federal Rural da Amazônia - UFRA, state of Pará, Brazil. The ecological indexes of parasitism were used according to Bush et al. (1997) and Bautista-Hernández et al. (2015). A review was made of the occurrence of Contracaecum larvae in different hosts present in the Brazilian ichthyofauna by means of databases, and the available results are shown in Table 1.

\section{Results}

A total of 150 nematodes were recovered from $A$. ocellatus showing prevalence: $67.5 \%$ (27/40), mean intensity: 5.5 , mean abundance: 3.7 and amplitude: 1 to 15 nematodes per fish. All specimens collected showed characteristics compatible with third stage larvae of Contracaecum sp. (Nematoda: Anisakidae). The parasites were encysted in the intestinal serosa and mesentery. The morphological and morphometric characteristics of the third-stage larvae of Contracaecum are presented below.

Contracaecum Railliet \& Henry, 1912

Contracaecum sp. (third-stage larvae) (Figs. 2 and 3)

(Description based on ten larvae)

Medium-sized nematodes, measuring $14 \mathrm{~mm}(11-16 \mathrm{~mm})$ in length, maximum width at ventriculus 451 (367-533), opaque-white when alive. Transversely striated cuticle present and more distinct at the extremities of the body, with anterior region interrupted by a short, lateral line (Figures 2a, 3a). Cephalic extremity rounded with a small, ventral cuticular tooth, 4 submedian cephalic papillae surrounding the small, transverse oval oral aperture; three poorly-developed lips (Figures 2b, 3b,c). Excretory pore situated below the ventral cephalic tooth (Figure 3b,c). Oesophageal muscle narrow, measuring $1.7(1-2 \mathrm{~mm}) \times 85(66-133)$ (Figure 2a). Ventricle is small, rounded, $86(66-120) \times 80(60-120)$; Ventricular appendix is short, $356(233-460) \times 76(66-93)$ (Figure 2c). Nerve 

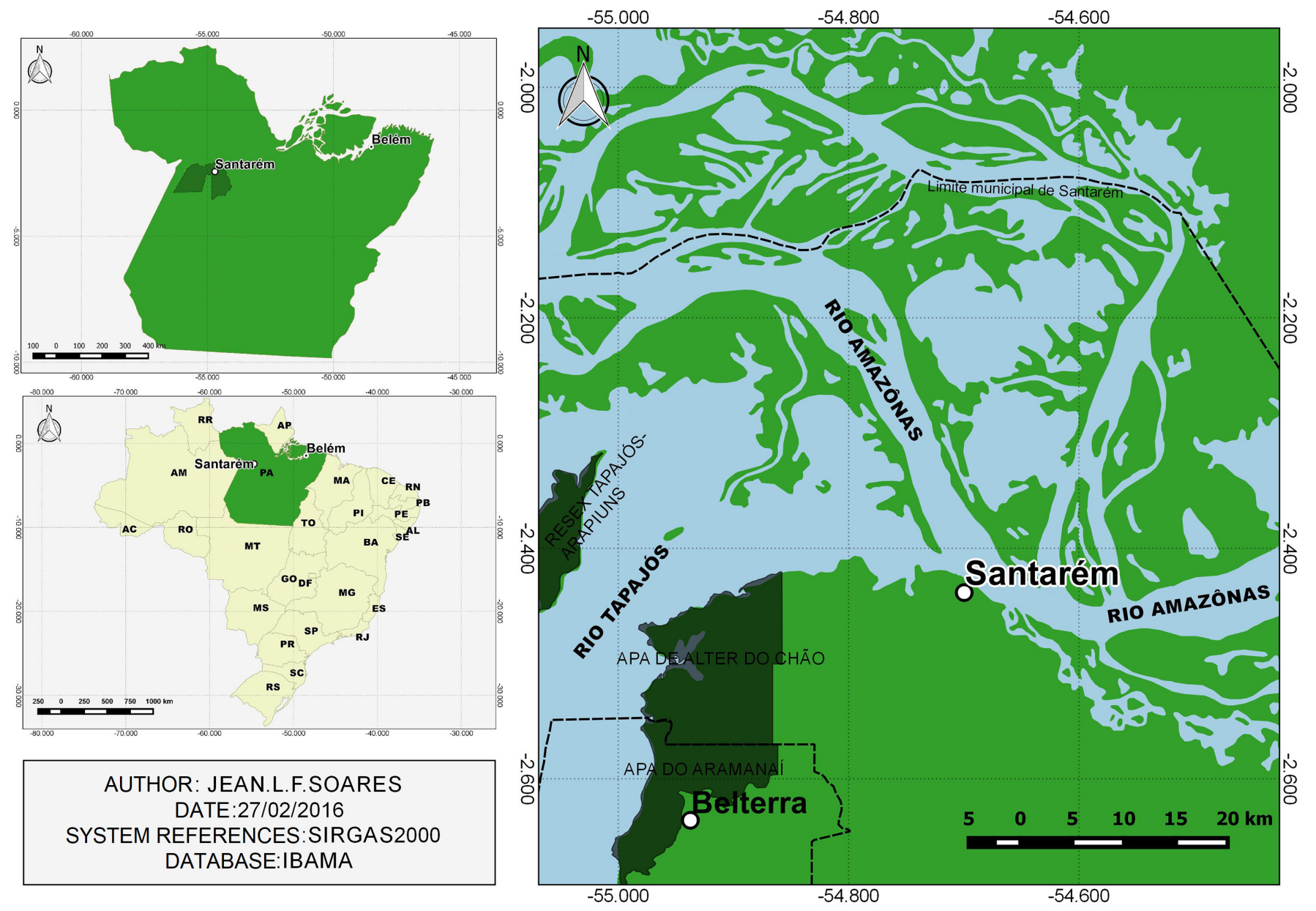

AUTHOR: JEAN.L.F.SOARES

DATE:27/02/2016

SYSTEM REFERENCES:SIRGAS2000

DATABASE:IBAMA

Figure 1. Collection locality of $A$. ocellatus in the Tapajós river, municipality of Santarém, State of Pará, Brazil.
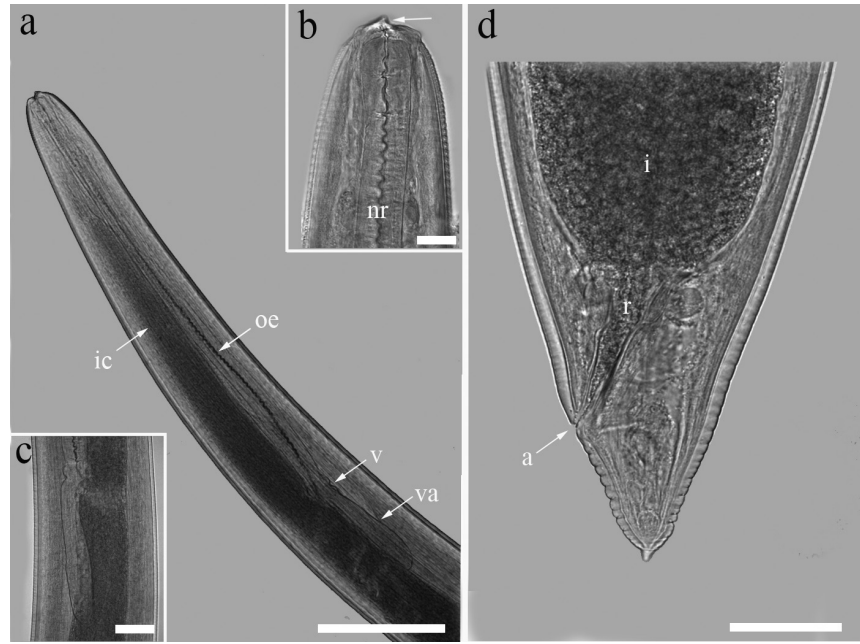

Figure 2. Light microscopy of Contracaecum sp. (L3 larvae) parasite of A. ocellatus: (a) Lateral view of the cephalic region showing evidence of the oesophagus (oe), intestinal caecum (ic), ventricle (v) and ventricular appendix (va). Bar $=200 \mu \mathrm{m}$; (b) Detail of larval tooth (arrow), nerve ring (nr) and cuticle with delicate transversal striations. Bar $=200 \mu \mathrm{m}$; (c) Detail of ventricle and ventricular appendix. Bar $=200 \mu \mathrm{m}$; (d) Posterior portion, portion of the intestine (i), rectum (r) and (a) anus, the tail without mucron. Bar $=50 \mu \mathrm{m}$. ring positioned at 283 (260-313) from the anterior extremity (Figure 2b). Deirids were observed only by SEM (Figure 3c). Long intestinal caecum, extending anteriorly to the nerve ring, measuring $1.2(1-1.5 \mathrm{~mm}) \times 145(120-167)$ (Figure 2a). The length ratio of the caecum and ventricular appendix is 30\% (20-40\%). Genital primordium is indistinct positioned at $4(3-5 \mathrm{~mm})$ from the anterior extremity. Rectum is a short hyaline tube; 3 small, unicellular rectal glands are present measuring 134 (110-233) (Figure 2d, 3e). Tail is conical, 131 (100-200) in length.

Taxonomic Summary:

Nematoda (Rudolphi, 1808)

Anisakidae Skrjabin \& Karokhin, 1945

Contracaecum sp. (L3 Larvae)

Host: Astronotus ocellatus (Agassiz, 1831) (Cichliformes: Cichlidae)

Common Name: acará-açu, apairi, oscar, bola de ouro and acará.

Length and mean weight of hosts: 19-29 (23) cm and 175-514 (354) g, respectively.

Infection sites: Encysted in the intestinal serosa and mesentery.

Biome: Amazon - Setting: freshwater

Locality type: River Tapajós, municipality of Santarém, Pará, eastern Brazilian Amazon. 
Table 1. Check list of records of third-stage larvae of Contracaecum spp. in fishes from Brazil.

\begin{tabular}{|c|c|c|c|}
\hline Order/ Family/ Host/ Environment ${ }^{\mathrm{a}}$ & $\begin{array}{c}\text { Site of } \\
\text { infection }^{b}\end{array}$ & Locality & Reference $^{d}$ \\
\hline \multicolumn{4}{|l|}{ Order Cichliformes } \\
\hline \multicolumn{4}{|l|}{ Family Cichlidae } \\
\hline Astronotus ocellatus (Agassiz, 1831) ${ }^{\mathrm{FW}}$ & ME, IN & $\begin{array}{l}\text { RJ, SP, PA, } \\
\text { AP }\end{array}$ & $\begin{array}{l}\text { Present study, Moravec (1998), Azevedo et al. } \\
\text { (2007; 2010; 2011), Eiras et al. (2010), Luque et al. } \\
\text { (2011), Neves et al. (2013), Tavares-Dias et al. } \\
\text { (2014), Bittencourt et al. (2014), Eiras et al. (2016), } \\
\text { Tavares-Dias \& Neves (2017) }\end{array}$ \\
\hline Geophagus brasiliensis (Quoy \& Gaimard, 1824) ${ }^{\mathrm{FW}, \mathrm{BW}}$ & $\begin{array}{l}\text { BC, ME, LI, } \\
\text { IN }\end{array}$ & $\begin{array}{l}\text { RJ, SP, PR, } \\
\text { RS }\end{array}$ & $\begin{array}{l}\text { Kohn et al. (1988), Luque \& Poulin (2004), } \\
\text { Paraguassú et al. (2005), Azevedo et al. (2006, } \\
\text { 2011), Eiras et al. (2010), Carvalho et al. (2010), } \\
\text { Luque et al. (2011), Bellay et al. (2012), Zago et al. } \\
\text { (2013), Rassier et al. (2015), Eiras et al. (2016) }\end{array}$ \\
\hline Cichla monoculus (Agassiz, 1831) $\mathrm{FW}$ & ME & PR & $\begin{array}{l}\text { Machado et al. (2000), Eiras et al. (2010), } \\
\text { Luque et al. (2011), Eiras et al. (2016) }\end{array}$ \\
\hline Cichla kelberi (Kullander \& FerrEiras, 2006) & $\mathrm{CO}, \mathrm{IN}$ & $\begin{array}{c}\text { PR, SP, RN, } \\
\text { MG }\end{array}$ & $\begin{array}{l}\text { Takemoto et al. (2009), Eiras et al. (2010), } \\
\text { Luque et al. (2011), Yamada \& Takemoto (2013), } \\
\text { Santos-Clapp \& Brasil-Sato (2014), Eiras et al. } \\
\text { (2016) }\end{array}$ \\
\hline Cichla piquiti (Kullander \& FerrEiras, 2006) ${ }^{\mathrm{FW}}$ & $\mathrm{BC}, \mathrm{ME}$ & SP, PR, TO & $\begin{array}{l}\text { Eiras et al. (2010), Franceschini et al. (2013b), } \\
\text { Yamada \& Takemoto (2013), Eiras et al. (2016) }\end{array}$ \\
\hline Cichla ocellaris (Bloch \& Schneider, 1801) FW, BW $^{\text {BW }}$ & $\begin{array}{l}\text { MU, BC, } \\
\text { ME, AC }\end{array}$ & SP, SE & $\begin{array}{l}\text { Martins et al. (2003), Eiras et al. (2010), Luque et al. } \\
\text { (2011), Eiras et al. (2016) }\end{array}$ \\
\hline Crenicichla lepidota $\left(\right.$ Heckel, 1840) ${ }^{\mathrm{FW}}$ & ST, PE, BC & PR & $\begin{array}{l}\text { Kohn et al. (1988), Moravec et al. (1993), Moravec } \\
\text { (1998), Vicente \& Pinto (1999), Eiras et al. (2010), } \\
\text { Luque et al. (2011), Eiras et al. (2016) }\end{array}$ \\
\hline Crenicichla britskii (Kullander, 1982) ${ }^{\mathrm{FW}}$ & IN & PR & Graça \& Machado (2007) \\
\hline Crenicichla haroldoi (Luengo \& Britski, 1974) FW $^{\mathrm{FW}}$ & NS & NS & Eiras et al. (2016) \\
\hline Oreochromis niloticus (Linnaeus, 1758) e, FW, BW $^{-}$ & IN & PR & Graça \& Machado (2007) \\
\hline \multicolumn{4}{|l|}{ Order Perciformes } \\
\hline \multicolumn{4}{|l|}{ Family Sciaenidae } \\
\hline Menticirrhus americanus (Linnaeus, 1758) $\mathrm{MAR}, \mathrm{BW}$ & IN & RJ & $\begin{array}{l}\text { Chaves \& Luque (1999), Luque et al. (2011), } \\
\text { Eiras et al. (2016) }\end{array}$ \\
\hline Micropogonias furnieri (Desmarest, 1823) & $\mathrm{ME}$ & RJ & $\begin{array}{l}\text { Alves \& Luque (2001a, b), Luque et al. (2010), } \\
\text { Luque et al. (2011), Mattos et al. (2014), Eiras et al. } \\
\text { (2016) }\end{array}$ \\
\hline Paralonchurus brasiliensis (Steindachner, 1875) ${ }^{\mathrm{MAR}, \mathrm{BW}}$ & $\mathrm{ME}$ & RJ & $\begin{array}{l}\text { Luque et al. (2003), Luque \& Poulin (2004), } \\
\text { Luque et al. (2011), Eiras et al. (2016) }\end{array}$ \\
\hline Pachyurus bonariensis (Steindachner, 1879) ${ }^{\mathrm{FW}}$ & ME & RJ, MT & $\begin{array}{l}\text { Lacerda et al. (2009), Eiras et al. (2010), Eiras et al. } \\
\text { (2016) }\end{array}$ \\
\hline Plagioscion squamosissimus (Heckel, 1840) ${ }^{\mathrm{FW}, \mathrm{BW}}$ & $\begin{array}{l}\text { ST, PE, } \\
\text { MU, ME, E, } \\
\text { LI, GO, AC, } \\
\text { G, GB, KI }\end{array}$ & PR, SP, PA & $\begin{array}{l}\text { Moravec et al. (1993), Moravec (1998), Vicente } \\
\text { \& Pinto (1999), Martins et al. (2003), Eiras et al. } \\
\text { (2010), Luque et al. (2011), Rodrigues et al. (2015), } \\
\text { Eiras et al. (2016) }\end{array}$ \\
\hline Plagioscion ternetzi (Boulenger, 1895) $\mathrm{FW}$ & ME & MT & $\begin{array}{l}\text { Luque et al. (2003), Lacerda et al. (2009), Eiras et al. } \\
\text { (2010), Eiras et al. (2016) }\end{array}$ \\
\hline Macrodon ancylodon (Bloch \& Schneider, 1801) & IN, ME & MA & $\begin{array}{l}\text { Vicente \& Fernandes (1978), Vicente et al. (1985), } \\
\text { Luque et al. (2011), Eiras et al. (2016) }\end{array}$ \\
\hline Cynoscion guatucupa (Cuvier, 1830) MAR & $\mathrm{ME}, \mathrm{AC}$ & $\mathrm{RJ}$ & Fontenelle et al. (2013) \\
\hline
\end{tabular}

abbreviations: Host species are given followed by their predominant habitat (MAR = marine, FW = freshwater or BW = brackish water); babbreviations: Site of the infection; when possibe these are grouped $(\mathrm{AC}=$ abdominal cavity; $\mathrm{BC}=$ body cavity; $\mathrm{CO}=$ coelom; $\mathrm{G}=\mathrm{Guts}$; $\mathrm{GI}=$ gills; $\mathrm{GO}=$ gonods; $\mathrm{GB}=$ gall baldder; $\mathrm{H}=$ heart; $\mathrm{HC}$ = hepatic capsule; $\mathrm{IN}$ = intestine; $\mathrm{IC}=$ intestinal cecum; $\mathrm{LI}=$ liver; $\mathrm{ME}=$ mesenteries; $\mathrm{Mu}=$ muscle; $\mathrm{SB}=$ swimming bladder; $\mathrm{PC}=$ pyloric caeca; $\mathrm{PE}=$ peritôneo; $\mathrm{ST}$ = stomach; $\mathrm{SV}=$ spiral valve; $\mathrm{KI}=$ Kidney; $\mathrm{GS}=$ gastric serosa; $\mathrm{NS}=$ not specified); clocalities are given in alphabetical order of Brazilian states (AM = Amazonas, $\mathrm{AP}=$ Amapá, $\mathrm{BA}=$ Bahia, $\mathrm{MA}=$ Maranhão, $\mathrm{MG}=$ Minas Gerais, $\mathrm{MS}=$ Mato Grosso do Sul, $\mathrm{MT}=$ Mato Grosso, $\mathrm{PA}=\mathrm{Pará}, \mathrm{PR}=\mathrm{Paraná}, \mathrm{RJ}=\mathrm{Rio}$ de Janeiro, RN = Rio Grande do Norte, RS = Rio Grande do Sul, SP = Sáo Paulo, SE = Sergipe, and TO = Tocantins); ${ }^{\mathrm{r}}$ records bibliographical by host in chronological sequence;

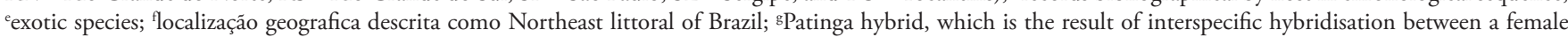

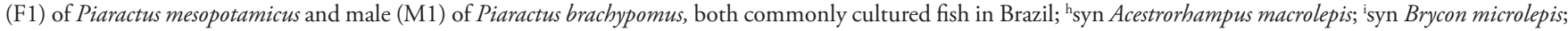
'syn Prochilodus scrofa; 'syn Paulicea luetkeni. 
Table 1. Continued...

\begin{tabular}{|c|c|c|c|}
\hline Order/ Family/ Host/ Environment ${ }^{a}$ & $\begin{array}{c}\text { Site of } \\
\text { infection }^{\mathrm{b}}\end{array}$ & Locality & Reference $^{d}$ \\
\hline Cynoscion striatus (Cuvier, 1829) ${ }^{\mathrm{MAR}}$ & NS & NS & Eiras et al. (2016) \\
\hline Cynoscion sp. ${ }^{\mathrm{MAR}}$ & NS & NS & Eiras et al. (2016) \\
\hline \multicolumn{4}{|l|}{ Family Carangidae } \\
\hline Caranx hippos (Linnaeus, 1766) $\mathrm{MAR}, \mathrm{BW}$ & $\mathrm{ME}$ & RJ & $\begin{array}{l}\text { Luque \& Poulin (2004), Luque et al. (2011), } \\
\text { Eiras et al. (2016) }\end{array}$ \\
\hline Caranx latus (Agassiz, 1831) & $\mathrm{ME}$ & RJ & $\begin{array}{l}\text { Luque \& Poulin (2004), Luque et al. (2011), } \\
\text { Eiras et al. (2016) }\end{array}$ \\
\hline Oligoplites palometa (Cuvier, 1832) ${ }^{\mathrm{MAR}, \mathrm{FW}, \mathrm{BW}}$ & GI & $\mathrm{RJ}$ & $\begin{array}{l}\text { Takemoto et al. (1996), Luque \& Poulin (2004), } \\
\text { Luque et al. (2011) }\end{array}$ \\
\hline Oligoplites saliens (Bloch, 1793) $\mathrm{MAR}, \mathrm{BW}$ & GI & RJ & $\begin{array}{l}\text { Takemoto et al. (1996), Luque \& Poulin (2004), } \\
\text { Luque et al. (2011) }\end{array}$ \\
\hline Oligoplites saurus (Bloch \& Schneider, 1801) ${ }^{\mathrm{MAR}, \mathrm{BW}}$ & GI & RJ & $\begin{array}{l}\text { Takemoto et al. (1996), Luque \& Poulin (2004), } \\
\text { Luque et al. (2011) }\end{array}$ \\
\hline Trachurus lathami (Nichols, 1920) & $\mathrm{ME}$ & RJ & Luque \& Poulin (2004), Luque et al. (2011) \\
\hline Selene setapinnis (Mitchill, 1815) $\mathrm{MAR}, \mathrm{BW}$ & ME & RJ & $\begin{array}{l}\text { Luque \& Poulin (2004), Cordeiro \& Luque (2004), } \\
\text { Luque et al. (2011) }\end{array}$ \\
\hline Parona signata (Jenyns, 1841) MAR & ME & NS & Luque et al (2011), Eiras et al. (2016) \\
\hline \multicolumn{4}{|l|}{ Family Scombridae } \\
\hline Scomber japonicus (Houttuyn, 1782) MAR & $\begin{array}{l}\mathrm{IC}, \mathrm{IN}, \mathrm{ME} \\
\mathrm{PC}, \mathrm{SB}\end{array}$ & RJ & $\begin{array}{l}\text { Rego \& Santos (1983), Vicente et al. (1985), } \\
\text { Alves et al. (2003), Luque et al. (2011) }\end{array}$ \\
\hline Euthynnus alletteratus (Rafinesque, 1810) ${ }^{\mathrm{MAR}, \mathrm{BW}}$ & $\mathrm{ME}$ & RJ & $\begin{array}{l}\text { Luque \& Poulin (2004), Alves \& Luque (2006), } \\
\text { Luque et al (2011), Eiras et al. (2016) }\end{array}$ \\
\hline Sarda sarda (Bloch, 1793) & ME & RJ & Alves \& Luque (2006), Luque et al. (2011) \\
\hline Scomberomorus cavalla (Cuvier, 1829) $)^{\mathrm{MAR}}$ & ME & RJ & Dias et al. (2011), Luque et al. (2011) \\
\hline $\begin{array}{l}\text { Scomberomorus brasiliensis (Collette, Russo \& Zavala- } \\
\text { Camin, 1978) }\end{array}$ & ME & RJ & Luque \& Poulin (2004), Luque et al. (2011) \\
\hline \multicolumn{4}{|l|}{ Family Sparidae } \\
\hline Pagrus pagrus (Linnaeus, 1758) MAR & $\begin{array}{l}\text { MU, LI, } \\
\text { ME, HC }\end{array}$ & RJ & $\begin{array}{l}\text { São Clemente et al. (1994), Barros (1994), } \\
\text { Paraguassú et al. (2002), Saad \& Luque (2009), } \\
\text { Luque et al. (2011), Soares et al. (2014), } \\
\text { Mattos et al. (2014), Eiras et al. (2016) }\end{array}$ \\
\hline Archosargus rhomboidalis (Linnaeus, 1758) $\mathrm{MAR}, \mathrm{BW}$ & NS & RJ & Luque \& Poulin (2004) \\
\hline \multicolumn{4}{|l|}{ Family Pomatomidae } \\
\hline Pomatomus saltatrix (Linnaeus, 1766) ${ }^{\mathrm{MAR}, \mathrm{BW}}$ & $\begin{array}{l}\text { ME, ST, LI, } \\
\mathrm{IC}, \mathrm{GO}, \mathrm{KI} \\
\mathrm{IN}, \mathrm{HC}, \mathrm{GS}\end{array}$ & RJ & $\begin{array}{l}\text { Rego et al. (1983), Vicente et al. (1985), Luque \& } \\
\text { Chaves (1999), Luque et al. (2011), Mattos et al. } \\
\text { (2014), Eiras et al. (2016) }\end{array}$ \\
\hline \multicolumn{4}{|l|}{ Family Mullidae } \\
\hline Mullus argentinae (Hubbs \& Marini, 1933) MAR & $\mathrm{ME}$ & RJ & $\begin{array}{l}\text { Luque et al. (2002), Luque \& Poulin (2004), } \\
\text { Luque et al. (2011), Eiras et al. (2016) }\end{array}$ \\
\hline \multicolumn{4}{|l|}{ Family Priacanthidae } \\
\hline Priacanthus arenatus (Cuvier, 1829) MAR & ME & RJ & $\begin{array}{l}\text { Tavares et al. (2001), Luque et al. (2011), Eiras et al. } \\
\text { (2016) }\end{array}$ \\
\hline \multicolumn{4}{|l|}{ Family Gerreidae } \\
\hline Diapterus rhombeus (Cuvier, 1829) $\mathrm{MAR}, \mathrm{BW}$ & ME & RJ & $\begin{array}{l}\text { Luque \& Poulin (2004), Luque et al. (2011), } \\
\text { Eiras et al. (2016) }\end{array}$ \\
\hline
\end{tabular}

abbreviations: Host species are given followed by their predominant habitat (MAR = marine, FW = freshwater or BW = brackish water); babbreviations: Site of the infection; when possibe these are grouped $(\mathrm{AC}=$ abdominal cavity; $\mathrm{BC}=$ body cavity; $\mathrm{CO}=$ coelom; $\mathrm{G}=\mathrm{Guts} ; \mathrm{GI}=$ gills; $\mathrm{GO}=$ gonods; $\mathrm{GB}=$ gall baldder; $\mathrm{H}=$ heart; $\mathrm{HC}$ = hepatic capsule; $\mathrm{IN}$ = intestine; $\mathrm{IC}=$ intestinal cecum; $\mathrm{LI}=$ liver; $\mathrm{ME}=$ mesenteries; $\mathrm{Mu}=$ muscle; $\mathrm{SB}=$ swimming bladder; $\mathrm{PC}=$ pyloric caeca; $\mathrm{PE}=$ peritôneo; $\mathrm{ST}$ = stomach; $\mathrm{SV}=$ spiral valve; $\mathrm{KI}=$ Kidney; GS = gastric serosa; NS = not specified); clocalities are given in alphabetical order of Brazilian states (AM = Amazonas, $\mathrm{AP}=$ Amapá, $\mathrm{BA}=$ Bahia, $\mathrm{MA}=$ Maranhão, $\mathrm{MG}=$ Minas Gerais, $\mathrm{MS}=$ Mato Grosso do Sul, $\mathrm{MT}=$ Mato Grosso, $\mathrm{PA}=\mathrm{Pará}, \mathrm{PR}=\mathrm{Paraná}, \mathrm{RJ}=\mathrm{Rio}$ de Janeiro,

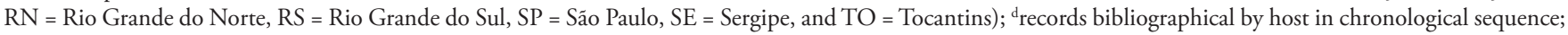
exotic species; flocalizaçấo geografica descrita como Northeast littoral of Brazil; sPatinga hybrid, which is the result of interspecific hybridisation between a female

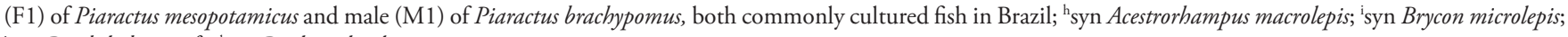
'syn Prochilodus scrofa; 'syn Paulicea luetkeni. 
Table 1. Continued...

\begin{tabular}{|c|c|c|c|}
\hline Order/ Family/ Host/ Environment ${ }^{a}$ & $\begin{array}{c}\text { Site of } \\
\text { infection }^{b}\end{array}$ & Locality & Reference $^{d}$ \\
\hline \multicolumn{4}{|l|}{ Family Trichiuridae } \\
\hline Trichiurus lepturus (Linnaeus, 1758) $\mathrm{MAR}, \mathrm{BW}$ & $\begin{array}{l}\mathrm{CO}, \mathrm{ME} \\
\mathrm{HC}\end{array}$ & RJ & $\begin{array}{l}\text { Barros \& Amato (1993), Silva et al. (2000), } \\
\text { Luque et al. (2011), Mattos et al. (2014) }\end{array}$ \\
\hline \multicolumn{4}{|l|}{ Family Centropomidae } \\
\hline Centropomus undecimalis (Bloch, 1792) ${ }^{\mathrm{MAR}, \mathrm{BW}, \mathrm{FW}}$ & $\mathrm{ME}$ & RJ, SP & $\begin{array}{l}\text { Luque \& Poulin (2004), Azevedo et al. (2011), } \\
\text { Luque et al. (2011), Eiras et al. (2016) }\end{array}$ \\
\hline \multicolumn{4}{|l|}{ Family Serranidae } \\
\hline Cephalopholis fulva (Linnaeus, 1758) & $\mathrm{ME}$ & RJ & $\begin{array}{l}\text { Luque \& Poulin (2004), Luque et al. (2011), } \\
\text { Eiras et al. (2016) }\end{array}$ \\
\hline \multicolumn{4}{|l|}{ Family Stromateidae } \\
\hline Peprilus paru (Linnaeus, 1758) $\mathrm{MAR}, \mathrm{BW}$ & NS & RJ & Luque \& Poulin (2004), Luque et al. (2011) \\
\hline \multicolumn{4}{|l|}{ Family Percophidae } \\
\hline Percophis brasiliensis (Quoy \& Gaimard, 1825) & ME & RJ & Luque \& Poulin (2004), Luque et al. (2011) \\
\hline \multicolumn{4}{|l|}{ Family Gempylidae } \\
\hline Thyrsitops lepidopoides (Cuvier, 1832) & NS & RJ & Domingues \& Alves (2015) \\
\hline \multicolumn{4}{|l|}{ Family Lutjanidae } \\
\hline Lutjanus purpureus (Poey, 1866) $\mathrm{MAR}$ & NS & f & Barros \& Cavalcanti (1998) \\
\hline Lutjanus synagris (Linnaeus, 1758) $\mathrm{MAR}$ & MU & RJ & Silva \& São Clemente (2001) \\
\hline \multicolumn{4}{|l|}{ Family Coryphaenidae } \\
\hline Coryphaena hippurus (Linnaeus, 1758) ${ }^{\mathrm{MAR}, \mathrm{BW}}$ & MU & RJ & Silva \& São Clemente (2001) \\
\hline \multicolumn{4}{|l|}{ Ordem Characiformes } \\
\hline \multicolumn{4}{|l|}{ Family Characidae } \\
\hline Astyanax fasciatus (Cuvier, 1819) $)^{\mathrm{FW}}$ & $\begin{array}{l}\text { ST, BC, } \\
\mathrm{ME}, \mathrm{AC}, \mathrm{LI} \\
\quad \text { IN }\end{array}$ & SP, MG & $\begin{array}{l}\text { Kloss (1966), Vicente et al. (1985), Moravec } \\
\text { (1998), Madi \& Silva (2005), Eiras et al. (2010), } \\
\text { Luque et al. (2011), Eiras et al. (2016), Vieira- } \\
\text { Menezes et al. (2017) }\end{array}$ \\
\hline Astyanax altiparanae (Garutti \& Britski, 2000) $)^{\mathrm{FW}}$ & $\begin{array}{c}\text { ME, IN, } \\
\text { BC, ME, IN }\end{array}$ & PR, SP & $\begin{array}{l}\text { Lizama et al. (2008), Takemoto et al. (2009), } \\
\text { Eiras et al. (2010), Luque et al. (2011), } \\
\text { Abdallah et al. (2012), Camargo et al. (2016a), } \\
\text { Eiras et al. (2016) }\end{array}$ \\
\hline Astyanax bockmanni (Vari \& Castro, 2007) $)^{\mathrm{FW}}$ & IN & SP & Camargo et al. (2016b) \\
\hline Astyanax bimaculatus (Linnaeus, 1758) ${ }^{\mathrm{FW}}$ & ST, BC, ME & SP & $\begin{array}{l}\text { Kloss (1966), Vicente et al. (1985), Moravec (1998), } \\
\text { Eiras et al. (2010), Luque et al. (2011), Eiras et al. } \\
\text { (2016) }\end{array}$ \\
\hline Astyanax schubarti (Britski, 1964) ${ }^{\mathrm{FW}}$ & ST, BC, ME & SP & $\begin{array}{l}\text { Kloss (1966), Vicente et al. (1985), Moravec (1998), } \\
\text { Eiras et al. (2010), Luque et al. (2011), Eiras et al. } \\
\text { (2016) }\end{array}$ \\
\hline Galeocharax humeralis (Valenciennes, 1834) ${ }^{\mathrm{FW}}$ & ST, PE, BC & PR & $\begin{array}{l}\text { Moravec et al. (1993), Moravec (1998), Vicente } \\
\text { \& Pinto (1999), Eiras et al. (2010), Luque et al. } \\
\text { (2011), Eiras et al. (2016) }\end{array}$ \\
\hline Galeocharax knerii (Steindachner, 1879) $)^{\mathrm{FW}}$ & ST, PE, BC & PR & $\begin{array}{l}\text { Vicente et al. (1985), Moravec et al. (1993), } \\
\text { Moravec (1998), Vicente \& Pinto (1999), Eiras et al. } \\
\text { (2010), Luque et al. (2011), Eiras et al. (2016) }\end{array}$ \\
\hline Hemibrycon surinamensis (Géry, 1962) FW $^{\mathrm{FW}}$ & IN & $\mathrm{AP}$ & Hoshino et al. (2014) \\
\hline Psellogrammus kennedyi (Eigenmann, 1903) FW $^{\mathrm{FW}}$ & NS & PR & $\begin{array}{l}\text { Takemoto et al. (2009), Eiras et al. (2010), } \\
\text { Luque et al. (2011), Eiras et al. (2016) }\end{array}$ \\
\hline Tetragonopterus argenteus (Cuvier, 1816) ${ }^{\mathrm{FW}}$ & NS & NS & Luque et al. (2011), Eiras et al. (2016) \\
\hline
\end{tabular}

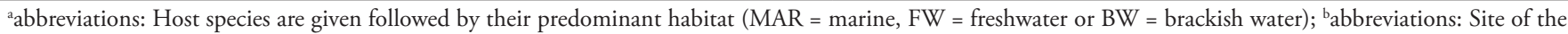
infection; when possibe these are grouped $(\mathrm{AC}=$ abdominal cavity; $\mathrm{BC}=$ body cavity; $\mathrm{CO}=$ coelom; $\mathrm{G}=\mathrm{Guts} ; \mathrm{GI}=$ gills; $\mathrm{GO}=$ gonods; $\mathrm{GB}=$ gall baldder; $\mathrm{H}=$ heart; $\mathrm{HC}=$ hepatic capsule; $\mathrm{IN}=$ intestine; $\mathrm{IC}=$ intestinal cecum; $\mathrm{LI}=$ liver; $\mathrm{ME}=$ mesenteries; $\mathrm{Mu}=$ muscle; $\mathrm{SB}=$ swimming bladder; $\mathrm{PC}=$ pyloric caeca; $\mathrm{PE}=$ peritôneo; $S T$ = stomach; $\mathrm{SV}=$ spiral valve; $\mathrm{KI}=$ Kidney; $\mathrm{GS}=$ gastric serosa; $\mathrm{NS}=$ not specified); clocalities are given in alphabetical order of Brazilian states (AM = Amazonas, $\mathrm{AP}=$ Amapá, $\mathrm{BA}=$ Bahia, $\mathrm{MA}=$ Maranhão, $\mathrm{MG}=$ Minas Gerais, $\mathrm{MS}=$ Mato Grosso do Sul, $\mathrm{MT}=$ Mato Grosso, $\mathrm{PA}=\mathrm{Pará}, \mathrm{PR}=\mathrm{Paraná}, \mathrm{RJ}=\mathrm{Rio}$ de Janeiro, RN = Rio Grande do Norte, RS = Rio Grande do Sul, SP = São Paulo, SE = Sergipe, and TO = Tocantins); drecords bibliographical by host in chronological sequence; exotic species; flocalização geografica descrita como Northeast littoral of Brazil; ${ }^{\mathrm{P}}$ Patinga hybrid, which is the result of interspecific hybridisation between a female

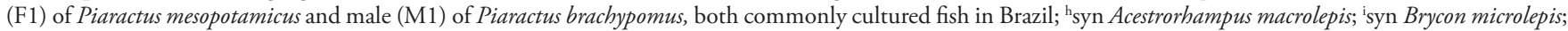
'syn Prochilodus scrofa; 'syn Paulicea luetkeni. 
Table 1. Continued...

\begin{tabular}{|c|c|c|c|}
\hline Order/ Family/ Host/ Environment ${ }^{\mathrm{a}}$ & $\begin{array}{c}\text { Site of } \\
\text { infection }^{\mathrm{b}}\end{array}$ & Locality & Reference $^{d}$ \\
\hline Markiana geayi (Pellegrin, 1909) ${ }^{\mathrm{FW}}$ & NS & NS & Eiras et al. (2016) \\
\hline Moenkhausia sanctaefilomenae (Steindachner, 1907) FW $^{\mathrm{FW}}$ & NS & NS & Eiras et al. (2016) \\
\hline Oligosarcus macrolepis (Steindachner, 1877) ${ }^{\mathrm{h}, \mathrm{FW}}$ & ST, PE, BC & PR & $\begin{array}{l}\text { Kohn et al. (1988), Vicente and Pinto (1999), } \\
\text { Eiras et al. (2010), Luque et al. (2011), Eiras et al. } \\
\text { (2016) }\end{array}$ \\
\hline Oligosarcus paranensis (Menezes \& Géry, 1983) FW $^{\mathrm{FW}}$ & $\mathrm{BC}$ & NS & $\begin{array}{l}\text { Eiras et al. (2010), Luque et al. (2011), Eiras et al. } \\
\text { (2016) }\end{array}$ \\
\hline Oligosarcus sp. ${ }^{\mathrm{FW}}$ & $\mathrm{BC}$ & SP & $\begin{array}{l}\text { Travassos et al. (1928), Eiras et al. (2010), } \\
\text { Luque et al. (2011), Eiras et al. (2016) }\end{array}$ \\
\hline \multicolumn{4}{|l|}{ Family Serrasalmidae } \\
\hline Metynnis lippincottianus (Cope, 1870) FW $^{\mathrm{FW}}$ & IN & PR, AP & $\begin{array}{l}\text { Moreira et al. (2009), Takemoto et al. (2009), } \\
\text { Eiras et al. (2010), Luque et al. (2011), Yamada et al. } \\
\text { (2012), Hoshino \& Tavares-Dias (2014), Eiras et al. } \\
\text { (2016) }\end{array}$ \\
\hline Metynnis hypsauchen (Müller \& Troschel, 1844) FW $^{\mathrm{FW}}$ & $\mathrm{AC}$ & $\mathrm{AP}$ & Oliveira et al. (2015) \\
\hline Serrasalmus marginatus (Valenciennes, 1837) ${ }^{\mathrm{FW}}$ & ME, ST, IC & $\begin{array}{l}\text { MT, MS, } \\
\quad \text { PR }\end{array}$ & $\begin{array}{l}\text { Barros et al. (2006), Vicentin et al. (2011), } \\
\text { Luque et al. (2011), Eiras et al. (2016), Casali \& } \\
\text { Takemoto (2016) }\end{array}$ \\
\hline Serrasalmus maculatus (Kner, 1858) FW $^{\mathrm{WW}}$ & $\mathrm{ME}$ & PR & Casali \& Takemoto (2016) \\
\hline Serrasalmus altuvei (Ramírez, 1965) $\mathrm{FW}$ & AM & & $\begin{array}{l}\text { Leão et al. (1991), Eiras et al. (2010), Luque et al. } \\
\text { (2011) }\end{array}$ \\
\hline Pygocentrus nattereri (Kner, 1858) ${ }^{\mathrm{FW}}$ & $\begin{array}{l}\text { BC, SB ME, } \\
\text { LI, ST, IC }\end{array}$ & MT, PA, MS & $\begin{array}{l}\text { Barros et al. (2006), Barros et al. (2010), Luque et al. } \\
\text { (2011), Benigno et al. (2012), Vicentin et al. (2013), } \\
\text { Eiras et al. (2016) }\end{array}$ \\
\hline Pygocentrus cariba (Humboldt, 1821) (HW $^{\mathrm{FW}}$ & $\mathrm{BC}$ & NS & Luque et al. (2011) \\
\hline Piaractus brachypomus (Cuvier, 1818) ${ }^{\mathrm{FW}}$ & IN, PC, AC & $\mathrm{AP}$ & Oliveira and Tavares-Dias (2016) \\
\hline Piaractus mesopotamicus (Holmberg, 1887) FW $^{\mathrm{FW}}$ & ME, BC & SP & Franceschini et al. (2013a) \\
\hline Patinga "Hybrid"g, Fw & $\mathrm{ME}, \mathrm{BC}$ & SP & Franceschini et al. (2013a) \\
\hline \multicolumn{4}{|l|}{ Family Anostomidae } \\
\hline Leporinus octofasciatus (Steindachner, 1915) ${ }^{\mathrm{FW}}$ & NS & SP & Madi \& Silva (2005), Eiras et al. (2010) \\
\hline Leporinus friderici (Bloch, 1794) ${ }^{\mathrm{FW}}$ & G, ME, IN & PR, AP & $\begin{array}{l}\text { Guidelli et al. (2006), Takemoto et al. (2009), } \\
\text { Eiras et al. (2010), Luque et al. (2011), } \\
\text { Guidelli et al. (2011), Eiras et al. (2016), } \\
\text { Oliveira et al. (2017), Yamada et al. (2017) }\end{array}$ \\
\hline Leporinus elongatus (Valenciennes, 1850) ${ }^{\mathrm{FW}}$ & G, ME, IN & PR & $\begin{array}{l}\text { Takemoto et al. (2009), Eiras et al. (2010), } \\
\text { Guidelli et al. (2011), Luque et al. (2011) }\end{array}$ \\
\hline Leporinus obtusidens (Valenciennes, 1837) ${ }^{\mathrm{FW}}$ & G, ME, IN & PR & $\begin{array}{l}\text { Takemoto et al. (2009), Eiras et al. (2010), } \\
\text { Luque et al. (2011), Guidelli et al. (2011) }\end{array}$ \\
\hline Leporinus lacustris (Amaral Campos, 1945) FW $^{\mathrm{FW}}$ & $\begin{array}{l}\text { BC, ME, G, } \\
\text { ME, IN }\end{array}$ & SP, PR & $\begin{array}{l}\text { Vicente et al. (1985), Moravec (1998), Guidelli et al. } \\
\text { (2006), Takemoto et al. (2009), Eiras et al. } \\
\text { (2010), Luque et al. (2011), Guidelli et al. (2011), } \\
\text { Eiras et al. (2016) }\end{array}$ \\
\hline Leporinus copelandii (Steindachner, 1875) & IN, BC & SP & $\begin{array}{l}\text { Vicente et al. (1985), Moravec (1998), Eiras et al. } \\
\text { (2010), Luque et al. (2011) }\end{array}$ \\
\hline Leporellus vittatus (Valenciennes, 1850) ${ }^{\mathrm{FW}}$ & $\mathrm{IN}, \mathrm{BC}$ & SP, PR & $\begin{array}{l}\text { Vicente et al. (1985), Moravec (1998), } \\
\text { Takemoto et al. (2009), Eiras et al. (2010), } \\
\text { Luque et al. (2011) }\end{array}$ \\
\hline
\end{tabular}

aabbreviations: Host species are given followed by their predominant habitat (MAR = marine, $\mathrm{FW}=$ freshwater or $\mathrm{BW}=$ brackish water); babbreviations: Site of the infection; when possibe these are grouped $(\mathrm{AC}=$ abdominal cavity; $\mathrm{BC}=$ body cavity; $\mathrm{CO}=$ coelom; $\mathrm{G}=\mathrm{Guts}$; $\mathrm{GI}=$ gills; $\mathrm{GO}=$ gonods; $\mathrm{GB}=$ gall baldder; $\mathrm{H}=$ heart; $\mathrm{HC}=$ hepatic capsule; $\mathrm{IN}$ = intestine; $\mathrm{IC}$ = intestinal cecum; $\mathrm{LI}=$ liver; $\mathrm{ME}=$ mesenteries; $\mathrm{Mu}=$ muscle; $\mathrm{SB}=$ swimming bladder; $\mathrm{PC}=$ pyloric caeca; $\mathrm{PE}$ = peritôneo; $\mathrm{ST}$ = stomach; $\mathrm{SV}$ = spiral valve; $\mathrm{KI}=$ Kidney; GS = gastric serosa; NS = not specified); clocalities are given in alphabetical order of Brazilian states (AM = Amazonas, $\mathrm{AP}=$ Amapá, $\mathrm{BA}=$ Bahia, $\mathrm{MA}=$ Maranhão, $\mathrm{MG}=$ Minas Gerais, $\mathrm{MS}=$ Mato Grosso do Sul, $\mathrm{MT}=$ Mato Grosso, $\mathrm{PA}=$ Pará, $\mathrm{PR}=\mathrm{Paraná}, \mathrm{RJ}=\mathrm{Rio} \mathrm{de} \mathrm{Janeiro,}$ RN = Rio Grande do Norte, RS = Rio Grande do Sul, SP = São Paulo, SE = Sergipe, and TO = Tocantins); ${ }^{\mathrm{d}}$ records bibliographical by host in chronological sequence;

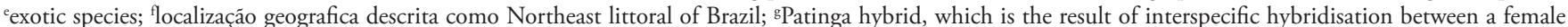
(F1) of Piaractus mesopotamicus and male (M1) of Piaractus brachypomus, both commonly cultured fish in Brazil; ${ }^{\mathrm{h}}$ syn Acestrorhampus macrolepis; ${ }^{\mathrm{i}}$ syn Brycon microlepis; 'syn Prochilodus scrofa; 'syn Paulicea luetkeni. 
Table 1. Continued...

\begin{tabular}{|c|c|c|c|}
\hline Order/ Family/ Host/ Environment ${ }^{a}$ & $\begin{array}{c}\text { Site of } \\
\text { infection }^{b}\end{array}$ & Locality & Reference $^{d}$ \\
\hline Hoplias malabaricus (Bloch, 1794) $)^{\mathrm{FW}}$ & $\begin{array}{l}\text { PE, G, CC, } \\
\text { SM, BC, } \\
\text { IN, LI, ST, } \\
\text { ME, MU, } \\
\text { IC, GO, } \\
\text { AC, IC }\end{array}$ & $\begin{array}{l}\text { RJ, PR, SP, } \\
\text { MT, PA, AP, } \\
\text { MA, RS }\end{array}$ & $\begin{array}{l}\text { Fábio (1982), Vicente et al. (1985), Weiblen \& } \\
\text { Brandâo (1992), Moravec et al. (1993), Moravec } \\
\text { (1998), Vicente \& Pinto (1999), Martins et al. } \\
\text { (2003), Barros et al. (2004), Madi \& Silva } \\
\text { (2005), Martins et al. (2005), Paraguassú \& } \\
\text { Luque (2007), Barros et al. (2007), Justino \& } \\
\text { Barros (2008), Takemoto et al. (2009), Eiras et al. } \\
\text { (2010), Luque et al. (2011), Benigno et al. (2012), } \\
\text { Alcântara \& Tavares-Dias (2015), Gonçalves et al. } \\
\text { (2016), Eiras et al. (2016), Rodrigues et al. (2017), } \\
\text { Carvalho et al. (2017) }\end{array}$ \\
\hline \multicolumn{4}{|l|}{ Family Acestrorhynchidae } \\
\hline Acestrorhynchus lacustris (Lütken, 1875) ${ }^{\mathrm{FW}}$ & $\begin{array}{l}\mathrm{H}, \mathrm{GO}, \mathrm{AC} \\
\text { SB, LI, E } \\
\text { S, IN }\end{array}$ & AM, PR, SP & $\begin{array}{l}\text { Carvalho et al. (2003), Takemoto et al. (2009), } \\
\text { Luque et al. (2011), Abdallah et al. (2012), } \\
\text { Camargo et al. (2015), Leite et al. (2017), } \\
\text { Pedro et al. (2016), Eiras et al. (2016) }\end{array}$ \\
\hline Acestrorhynchus falcatus (Bloch, 1794) ${ }^{\mathrm{FW}}$ & IN, LI & $\mathrm{AP}$ & Hoshino et al. (2016) \\
\hline Acestrorhynchus falcirostris (Cuvier, 1819) ${ }^{\mathrm{FW}}$ & IN, LI & $\mathrm{AP}$ & Hoshino et al. (2016) \\
\hline \multicolumn{4}{|l|}{ Family Bryconidae } \\
\hline Salminus hilarii (Valenciennes, 1850) ${ }^{\mathrm{FW}}$ & $\mathrm{ME}$ & SP & $\begin{array}{l}\text { Madi \& Silva (2005), Eiras et al. (2010), } \\
\text { Luque et al. (2011) }\end{array}$ \\
\hline Salminus brasiliensis (Cuvier, 1816) ${ }^{\mathrm{FW}}$ & BC, ME, IN & SP, PR & $\begin{array}{l}\text { Vicente et al. (1985), Moravec (1998), Eiras et al. } \\
\text { (2010), Luque et al. (2011), Mesquita et al. (2012), } \\
\text { Karling et al. (2013a), Eiras et al. (2016) }\end{array}$ \\
\hline Rhaphiodon vulpinus (Spix \& Agassiz, 1829) ${ }^{\mathrm{FW}}$ & $\mathrm{ME}$ & PR, MS & $\begin{array}{l}\text { Moravec et al. (1993), Moravec (1998), Vicente \& } \\
\text { Pinto (1999), Takemoto et al. (2009), Eiras et al. } \\
\text { (2010), Luque et al. (2011), Karling et al. (2013b), } \\
\text { Eiras et al. (2016) }\end{array}$ \\
\hline \multicolumn{4}{|l|}{ Family Triportheidae } \\
\hline Triportheus angulatus (Spix \& Agassiz, 1829) & IN & AP, SP & Abdallah et al. (2012), Oliveira et al. (2016) \\
\hline \multicolumn{4}{|l|}{ Family Curimatidae } \\
\hline Cyphocharax modestus (Fernández-Yépez, 1948) & LI & SP & Abdallah et al. (2012), Eiras et al. (2016) \\
\hline Cyphocharax nagelii (Steindachner, 1881) ${ }^{\mathrm{FW}}$ & IN, ST & SP & $\begin{array}{l}\text { Abdallah et al. (2012), Vieira et al. (2013), } \\
\text { Eiras et al. (2016) }\end{array}$ \\
\hline
\end{tabular}

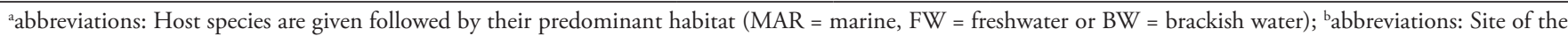
infection; when possibe these are grouped $(\mathrm{AC}=$ abdominal cavity; $\mathrm{BC}=$ body cavity; $\mathrm{CO}=$ coelom; $\mathrm{G}=\mathrm{Guts} ; \mathrm{GI}=$ gills; $\mathrm{GO}=$ gonods; $\mathrm{GB}=$ gall baldder; $\mathrm{H}=$ heart; $\mathrm{HC}=$ hepatic capsule; $\mathrm{IN}=$ intestine; $\mathrm{IC}=$ intestinal cecum; $\mathrm{LI}=$ liver; $\mathrm{ME}=$ mesenteries; $\mathrm{Mu}=$ muscle; $\mathrm{SB}=$ swimming bladder; $\mathrm{PC}=$ pyloric caeca; $\mathrm{PE}=$ peritôneo; $\mathrm{ST}$ = stomach; $\mathrm{SV}=$ spiral valve; $\mathrm{KI}=$ Kidney; $\mathrm{GS}=$ gastric serosa; $\mathrm{NS}=$ not specified); clocalities are given in alphabetical order of Brazilian states (AM = Amazonas, $\mathrm{AP}=$ Amapá, $\mathrm{BA}=$ Bahia, $\mathrm{MA}=$ Maranhão, $\mathrm{MG}=$ Minas Gerais, $\mathrm{MS}=$ Mato Grosso do Sul, $\mathrm{MT}=$ Mato Grosso, $\mathrm{PA}=\mathrm{Pará}, \mathrm{PR}=\mathrm{Paraná}, \mathrm{RJ}=\mathrm{Rio}$ de Janeiro, $\mathrm{RN}=$ Rio Grande do Norte, RS = Rio Grande do Sul, SP = São Paulo, SE = Sergipe, and TO = Tocantins); ${ }^{\mathrm{d}}$ records bibliographical by host in chronological sequence; exotic species; 'localização geografica descrita como Northeast littoral of Brazil; ${ }^{8}$ Patinga hybrid, which is the result of interspecific hybridisation between a female (F1) of Piaractus mesopotamicus and male (M1) of Piaractus brachypomus, both commonly cultured fish in Brazil; ' ${ }^{\mathrm{h}}$ syn Acestrorhampus macrolepis; ; syn Brycon microlepis; 'syn Prochilodus scrofa; 'syn Paulicea luetkeni. 
Table 1. Continued...

\begin{tabular}{lccl}
\hline \multicolumn{1}{c}{ Order/ Family/ Host/ Environment } & $\begin{array}{c}\text { Site of } \\
\text { infection }^{\mathbf{b}}\end{array}$ & Locality $^{\mathbf{c}}$ & Reference $^{\mathbf{d}}$ \\
\hline Curimata sp. ${ }^{\mathrm{FW}}$ & BC & SP & $\begin{array}{l}\text { Vicente et al. (1985), Moravec (1998), Eiras et al. } \\
\text { (2010), Luque et al. (2011) }\end{array}$ \\
$\begin{array}{l}\text { Family Prochilodontidae } \\
\text { Prochilodus lineatus (Valenciennes, 1837), FW }\end{array}$ & IN, CA, PE, & SP, PR & $\begin{array}{l}\text { Vicente et al. (1985), Moravec et al. (1993), } \\
\text { Moravec (1998), Vicente \& Pinto (1999), Eiras et al. } \\
\text { (2010), Eiras et al. (2016) }\end{array}$ \\
Ordem Siluriformes & ST & &
\end{tabular}

\section{Ordem Siluriformes \\ Family Pimelodidae}

Hemisorubim platyrhynchos (Valenciennes, 1840) FW $^{\mathrm{FW}}$

Pseudoplatystoma fasciatum (Linnaeus, 1766) FW $^{\mathrm{FW}}$

Pseudoplatystoma corruscans (Spix \& Agassiz, 1829) ${ }^{\mathrm{FW}}$

Brachyplatystoma filamentosum (Lichtenstein, 1819) ${ }^{\mathrm{FW}, \mathrm{BW}}$

Brachyplatystoma rousseauxii (Castelnau, 1855) FW $^{\mathrm{FW}}$

Pinirampus pirinampu (Spix \& Agassiz, 1829) $)^{\mathrm{FW}}$

Zungaro zungaro (Humboldt, 1821) 1, FW $^{\mathrm{FW}}$

Iheringichthys labrosus (Lütken, 1874) FW $^{\mathrm{FW}}$

Pimelodus maculatus (Lacepède, 1803) FW $^{\mathrm{FW}}$

Pimelodus ortmanni (Haseman, 1911) FW $^{\mathrm{FW}}$

Pimelodus pohli (Ribeiro \& Lucena, 2006) FW $^{\mathrm{FW}}$

Bergiaria sp. ${ }^{\mathrm{FW}}$

\section{Family Auchenipteridae}

\begin{tabular}{|c|c|c|c|}
\hline Trachelyopterus striatulus (Steindachner, 1877) ${ }^{\mathrm{FW}}$ & LI & RJ & Azevedo et al. (2011), Luque et al. (2011) \\
\hline Trachelyopterus galeatus (Linnaeus, 1766) ${ }^{\mathrm{FW}}$ & IN & $\mathrm{AP}$ & Pantoja et al. (2016) \\
\hline Trachelyopterus coriaceus (Valenciennes, 1840) ${ }^{\mathrm{FW}}$ & IN & $\mathrm{AP}$ & Pantoja et al. (2016) \\
\hline Auchenipterus osteomystax (Miranda Ribeiro, 1918) FW & NS & NS & Eiras et al. (2016) \\
\hline
\end{tabular}

a abbreviations: Host species are given followed by their predominant habitat (MAR = marine, FW = freshwater or BW = brackish water); babbreviations: Site of the infection; when possibe these are grouped $(\mathrm{AC}=$ abdominal cavity; $\mathrm{BC}=$ body cavity; $\mathrm{CO}=$ coelom; $\mathrm{G}=\mathrm{Guts} ; \mathrm{GI}=$ gills; $\mathrm{GO}=$ gonods; $\mathrm{GB}=$ gall baldder; $\mathrm{H}=$ heart; $\mathrm{HC}$ = hepatic capsule; $\mathrm{IN}=$ intestine; $\mathrm{IC}=$ intestinal cecum; $\mathrm{LI}=$ liver; $\mathrm{ME}=$ mesenteries; $\mathrm{Mu}=$ muscle; $\mathrm{SB}=$ swimming bladder; $\mathrm{PC}=$ pyloric caeca; $\mathrm{PE}=$ peritôneo; $\mathrm{ST}$ = stomach; $\mathrm{SV}$ = spiral valve; $\mathrm{KI}=$ Kidney; GS = gastric serosa; NS = not specified); clocalities are given in alphabetical order of Brazilian states (AM = Amazonas, $\mathrm{AP}=$ Amapá, $\mathrm{BA}=$ Bahia, $\mathrm{MA}=$ Maranhão, $\mathrm{MG}=$ Minas Gerais, $\mathrm{MS}=$ Mato Grosso do Sul, $\mathrm{MT}=$ Mato Grosso, $\mathrm{PA}=\mathrm{Pará}, \mathrm{PR}=\mathrm{Paraná}, \mathrm{RJ}=\mathrm{Rio}$ de Janeiro, $\mathrm{RN}=$ Rio Grande do Norte, RS = Rio Grande do Sul, SP = São Paulo, SE = Sergipe, and TO = Tocantins); ${ }^{\mathrm{d}}$ records bibliographical by host in chronological sequence; exotic species; ' localizaçấo geografica descrita como Northeast littoral of Brazil; sPatinga hybrid, which is the result of interspecific hybridisation between a female

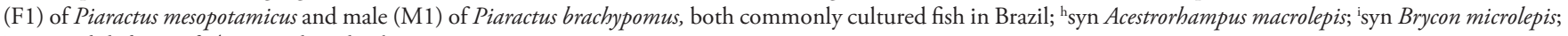

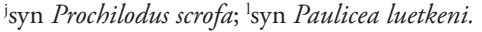

\begin{tabular}{|c|c|c|}
\hline $\begin{array}{l}\text { ME, E, LI, } \\
\text { GO, AC, }\end{array}$ & PA & Rodrigues et al. (2015) \\
\hline \multicolumn{3}{|l|}{ GU, GB, KI } \\
\hline $\begin{array}{l}\text { ME, E, LI, } \\
\text { GO, AC, }\end{array}$ & PA & Rodrigues et al. (2015) \\
\hline \multicolumn{3}{|l|}{ GU, GB, KI } \\
\hline ME & MT & $\begin{array}{l}\text { Barros et al. (2006), Luque et al. (2011), Eiras et al. } \\
\text { (2016) }\end{array}$ \\
\hline ME & MT & $\begin{array}{l}\text { Barros et al. (2006), Luque et al. (2011), Eiras et al. } \\
\text { (2016) }\end{array}$ \\
\hline IN & PR & $\begin{array}{l}\text { Moreira et al. (2005), Takemoto et al. (2009), } \\
\text { Eiras et al. (2010), Luque et al. (2011), Eiras et al. } \\
\text { (2016) }\end{array}$ \\
\hline ME & PR & $\begin{array}{l}\text { Takemoto et al. (2009), Eiras et al. (2010), } \\
\text { Luque et al. (2011) }\end{array}$ \\
\hline ST, PE, BC & PR & $\begin{array}{l}\text { Kohn et al. (1988), Vicente \& Pinto (1999), } \\
\text { Eiras et al. (2010), Luque et al. (2011), Eiras et al. } \\
(2016)\end{array}$ \\
\hline $\mathrm{CO}$ & MG & Sabas \& Brasil-Sato (2014), Eiras et al. (2016) \\
\hline ST, PE, BC & PR & $\begin{array}{l}\text { Kohn et al. (1988), Vicente \& Pinto (1999), } \\
\text { Eiras et al. (2010), Luque et al. (2011), Eiras et al. } \\
(2016)\end{array}$ \\
\hline LI & RJ & Azevedo et al. (2011), Luque et al. (2011) \\
\hline IN & $\mathrm{AP}$ & Pantoja et al. (2016) \\
\hline IN & $\mathrm{AP}$ & Pantoja et al. (2016) \\
\hline NS & NS & Eiras et al. (2016) \\
\hline \multicolumn{3}{|c|}{ 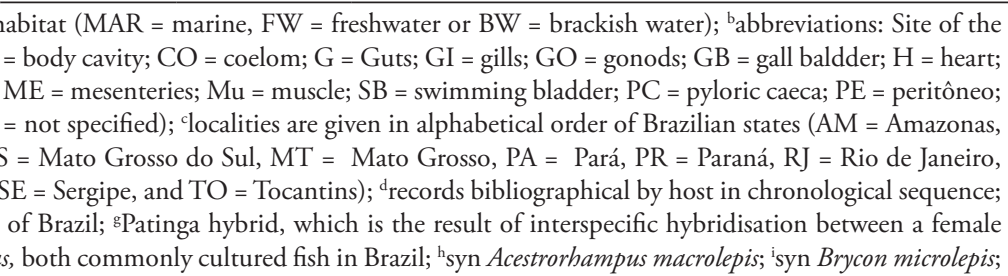 } \\
\hline
\end{tabular}


Table 1. Continued...

\begin{tabular}{|c|c|c|c|}
\hline Order/ Family/ Host/ Environment ${ }^{a}$ & $\begin{array}{c}\text { Site of } \\
\text { infection }^{\mathrm{b}}\end{array}$ & Locality & Reference $^{\mathrm{d}}$ \\
\hline Ageneiosus inermis (Linnaeus, 1766) FW $^{\mathrm{FW}}$ & NS & NS & Eiras et al. (2010), Luque et al. (2011) \\
\hline Ageneiosus ucayalensis Castelnau, $1855^{\mathrm{FW}}$ & IN, CA & $\mathrm{AP}$ & Ferreira \& Tavares-Dias (2017) \\
\hline \multicolumn{4}{|l|}{ Family Heptapteridae } \\
\hline Rhamdia quelen (Quoy \& Gaimard, 1824) & $\begin{array}{l}\text { ME, ST, } \\
\text { IN, LI }\end{array}$ & SP, RS & $\begin{array}{l}\text { Kohn et al. (1989), Madi \& Silva (2005), Eiras et al. } \\
\text { (2010), Azevedo et al. (2011), Luque et al. (2011), } \\
\text { Dias et al. (2016), Eiras et al. (2016) }\end{array}$ \\
\hline Pimelodella lateristriga (Lichtenstein, 1823) $)^{\mathrm{FW}}$ & NS & SP & $\begin{array}{l}\text { Travassos et al. (1928), Eiras et al. (2010), } \\
\text { Luque et al. (2011) }\end{array}$ \\
\hline \multicolumn{4}{|l|}{ Family Doradidae } \\
\hline Oxydoras niger (Valenciennes, 1821) ${ }^{\mathrm{FW}}$ & $\begin{array}{l}\text { ME, ST, LI, } \\
\text { GO, AC, G, } \\
\text { GB, KI }\end{array}$ & PA & Rodrigues et al. (2015) \\
\hline \multicolumn{4}{|l|}{ Family Ariidae } \\
\hline Genidens barbus (Lacepède, 1803) ${ }^{\mathrm{MAR}, \mathrm{BW}}$ & $\mathrm{ME}$ & RJ & $\begin{array}{l}\text { Tavares \& Luque (2004), Luque \& Poulin (2004), } \\
\text { Luque et al. (2011), Eiras et al. (2016) }\end{array}$ \\
\hline \multicolumn{4}{|l|}{ Family Loricariidae } \\
\hline Loricariichthys castaneus (Castelnau, 1855) ${ }^{\mathrm{FW}}$ & ME & SP & $\begin{array}{l}\text { Azevedo et al. (2011), Luque et al. (2011), } \\
\text { Eiras et al. (2016) }\end{array}$ \\
\hline \multicolumn{4}{|l|}{ Ordem Carcharhiniformes } \\
\hline \multicolumn{4}{|l|}{ Family Carcharhinidae } \\
\hline Carcharhinus brachyurus (Günther, 1870) & SV & RS & $\begin{array}{l}\text { Knoff et al. (2001), Luque et al. (2011), Eiras et al. } \\
(2016)\end{array}$ \\
\hline Carcharhinus signatus (Poey, 1868) ${ }^{\mathrm{MAR}}$ & SV, ST & PR & $\begin{array}{l}\text { Knoff et al. (2001), Luque et al. (2011), Eiras et al. } \\
(2016)\end{array}$ \\
\hline \multicolumn{4}{|l|}{ Family Triakidae } \\
\hline Galeorhinus galeus (Linnaeus, 1758) & SV & RS & $\begin{array}{l}\text { Knoff et al. (2001), Luque et al. (2011), Eiras et al. } \\
\text { (2016) }\end{array}$ \\
\hline Mustelus canis (Mitchill, 1815) ${ }^{\mathrm{MAR}, \mathrm{BW}}$ & SV, ST & RS & Knoff et al. (2001), Luque et al. (2011) \\
\hline Mustelus schmitti (Springer, 1939) & SV & RS & Knoff et al. (2001), Luque et al. (2011) \\
\hline \multicolumn{4}{|l|}{ Family Scyliorhinidae } \\
\hline Scyliorhinus haeckelii (Miranda Ribeiro, 1907) ${ }^{\mathrm{MAR}}$ & SV, ST & PR & Knoff et al. (2001), Luque et al. (2011) \\
\hline \multicolumn{4}{|l|}{ Family Sphyrnidae } \\
\hline Sphyrna zygaena (Linnaeus, 1758) MAR, BW & SV, ST & RS & Knoff et al. (2001), Luque et al. (2011) \\
\hline \multicolumn{4}{|l|}{ Ordem Tetraodontiformes } \\
\hline \multicolumn{4}{|l|}{ Family Balistidae } \\
\hline Balistes capriscus (Gmelin, 1789) MAR & $\mathrm{ME}$ & RJ & $\begin{array}{l}\text { Luque \& Poulin (2004), Alves et al. (2005), } \\
\text { Luque et al. (2011), Eiras et al. (2016) }\end{array}$ \\
\hline Balistes vetula (Linnaeus, 1758) & ME & RJ & $\begin{array}{l}\text { Luque \& Poulin (2004), Alves et al. (2005), } \\
\text { Luque et al. (2011), Eiras et al. (2016) }\end{array}$ \\
\hline \multicolumn{4}{|l|}{ Family Monacanthidae } \\
\hline Aluterus monoceros (Linnaeus, 1758) ${ }^{\mathrm{MAR}}$ & LI, ME & RJ & $\begin{array}{l}\text { Luque \& Poulin (2004), Dias et al. (2010), } \\
\text { Luque et al. (2011), Eiras et al. (2016) }\end{array}$ \\
\hline \multicolumn{4}{|l|}{ Ordem Clupeiformes } \\
\hline \multicolumn{4}{|l|}{ Family Clupeidae } \\
\hline Harengula clupeola (Cuvier, 1829) $\mathrm{MAR}$ & NS & BA & Guimarães \& Cristofaro (1974) \\
\hline
\end{tabular}

abbreviations: Host species are given followed by their predominant habitat (MAR = marine, FW = freshwater or BW = brackish water); ${ }^{b}$ abbreviations: Site of the infection; when possibe these are grouped $(\mathrm{AC}=$ abdominal cavity; $\mathrm{BC}=$ body cavity; $\mathrm{CO}=$ coelom; $\mathrm{G}=\mathrm{Guts}$; $\mathrm{GI}$ = gills; $\mathrm{GO}=$ gonods; $\mathrm{GB}=$ gall baldder; $\mathrm{H}=$ heart; $\mathrm{HC}=$ hepatic capsule; $\mathrm{IN}$ = intestine; $\mathrm{IC}=$ intestinal cecum; $\mathrm{LI}=$ liver; $\mathrm{ME}=$ mesenteries; $\mathrm{Mu}=$ muscle; $\mathrm{SB}=$ swimming bladder; $\mathrm{PC}=$ pyloric caeca; $\mathrm{PE}=$ peritôneo; ST = stomach; SV = spiral valve; KI = Kidney; GS = gastric serosa; NS = not specified); clocalities are given in alphabetical order of Brazilian states (AM = Amazonas, $\mathrm{AP}=$ Amapá, $\mathrm{BA}=$ Bahia, $\mathrm{MA}=$ Maranhão, $\mathrm{MG}=$ Minas Gerais, $\mathrm{MS}=$ Mato Grosso do Sul, $\mathrm{MT}=$ Mato Grosso, $\mathrm{PA}=$ Pará, $\mathrm{PR}=\mathrm{Paraná}$, $\mathrm{RJ}=\mathrm{Rio}$ de Janeiro,

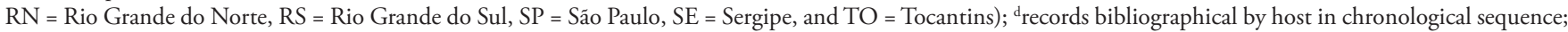
'exotic species; flocalização geografica descrita como Northeast littoral of Brazil; sPatinga hybrid, which is the result of interspecific hybridisation between a female

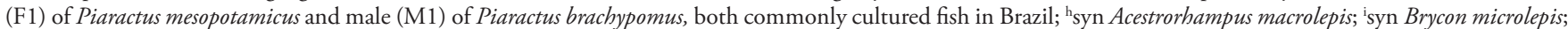
'syn Prochilodus scrofa; 'syn Paulicea luetkeni. 
Table 1. Continued...

\begin{tabular}{|c|c|c|c|}
\hline Order/ Family/ Host/ Environment ${ }^{a}$ & $\begin{array}{c}\text { Site of } \\
\text { infection }^{\text {b }}\end{array}$ & Locality & Reference $^{d}$ \\
\hline Brevoortia aurea (Spix \& Agassiz, 1829) & $\mathrm{ME}$ & $\mathrm{RJ}$ & $\begin{array}{l}\text { Luque \& Poulin (2004), Luque et al. (2011), } \\
\text { Eiras et al. (2016) }\end{array}$ \\
\hline \multicolumn{4}{|l|}{ Ordem Scorpaeniformes } \\
\hline \multicolumn{4}{|l|}{ Family Dactylopteridae } \\
\hline Dactylopterus volitans (Linnaeus, 1758) & ME & RJ & $\begin{array}{l}\text { Luque \& Poulin (2004), Cordeiro \& Luque (2005), } \\
\text { Luque et al. (2011), Eiras et al. (2016) }\end{array}$ \\
\hline
\end{tabular}

\section{Ordem Gymnotiformes}

Family Gymnotidae

Gymnotus carapo (Linnaeus, 1758) ${ }^{\mathrm{FW}}$

Gymnotus sp. ${ }^{\mathrm{FW}}$

\section{Ordem Gadiformes}

\section{Family Phycidae}

Urophycis mystacea (Miranda Ribeiro, 1903) MAR

Urophycis brasiliensis (Kaup, 1858) $\mathrm{MAR}$

\section{Ordem Lophiiformes}

\section{Family Lophiidae}

Lophius gastrophysus (Miranda Ribeiro, 1915) MAR

Ordem Ophidiiformes

Family Ophidiidae

Genypterus brasiliensis (Regan, 1903) MAR

Genypterus blacodes (Forster, 1801) MAR

Ordem Pleuronectiformes

Family Paralichthyidae

Paralichthys isosceles (Jordan, 1891) MAR

Paralichthys patagonicus (Jordan, 1889) MAR

Xystreurys rasile (Jordan, 1891) $\mathrm{MAR}$

\section{Ordem Rajiformes}

\section{Family Rajidae}

Dipturus trachyderma (Krefft \& Stehmann, 1975) MAR

Ordem Hexanchiformes

\section{Family Hexanchidae}

Heptranchias perlo (Bonnaterre, 1788) ${ }^{\mathrm{MAR}}$

Hexanchus griseus (Bonnaterre, 1788) $\mathrm{MAR}$

$\mathrm{ME}$
SV, ST PR Knoff et al. (2001), Luque et al. (2011), Eiras et al.

ST, ME, IN SP, RJ

MS

ME $\quad$ RJ Luque et al. (2011)

ME RJ Alves et al. (2004), Luque et al. (2011)

ME RJ Saad et al. (2012), Eiras et al. (2016)

ME RJ

NS NS

Alves et al. (2002), Knoff et al. (2007), Luque et al. (2011), Mattos et al. (2014)

Eiras et al. (2016)

ME, IN RJ Luque \& Poulin (2004), Felizardo et al. (2009), Luque et al. (2011), Alarcos et al. (2016), Eiras et al. (2016)

MU, ST, RJ Fonseca et al. (2016)

IN, AC

ST, IN, AC RJ Fonseca et al. (2016) (2016)

SV PR

Knoff et al. (2001), Luque et al. (2011), Eiras et al. (2016)

SV $\quad$ PR

\section{Ordem Squatiniformes}

\section{Family Squatinidae}

Squatina sp. MAR

a abbreviations: Host species are given followed by their predominant habitat (MAR = marine, $\mathrm{FW}=$ freshwater or BW = brackish water); babbreviations: Site of the infection; when possibe these are grouped $(\mathrm{AC}=$ abdominal cavity; $\mathrm{BC}=$ body cavity; $\mathrm{CO}=$ coelom; $\mathrm{G}=\mathrm{Guts} ; \mathrm{GI}=$ gills; $\mathrm{GO}=$ gonods; $\mathrm{GB}=$ gall baldder; $\mathrm{H}=$ heart; $\mathrm{HC}=$ hepatic capsule; $\mathrm{IN}$ = intestine; $\mathrm{IC}=$ intestinal cecum; $\mathrm{LI}=$ liver; $\mathrm{ME}=$ mesenteries; $\mathrm{Mu}=$ muscle; $\mathrm{SB}=$ swimming bladder; $\mathrm{PC}=$ pyloric caeca; $\mathrm{PE}=$ peritôneo; $\mathrm{ST}=$ stomach; $\mathrm{SV}=$ spiral valve; $\mathrm{KI}=$ Kidney; $\mathrm{GS}=$ gastric serosa; $\mathrm{NS}=$ not specified); clocalities are given in alphabetical order of Brazilian states (AM = Amazonas, $\mathrm{AP}=$ Amapá, $\mathrm{BA}=$ Bahia, $\mathrm{MA}=$ Maranhão, $\mathrm{MG}=$ Minas Gerais, $\mathrm{MS}=$ Mato Grosso do Sul, $\mathrm{MT}=$ Mato Grosso, $\mathrm{PA}=$ Pará, $\mathrm{PR}=\mathrm{Paraná}, \mathrm{RJ}=\mathrm{Rio} \mathrm{de} \mathrm{Janeiro,}$ RN = Rio Grande do Norte, RS = Rio Grande do Sul, SP = São Paulo, SE = Sergipe, and TO = Tocantins); ${ }^{\mathrm{d}}$ records bibliographical by host in chronological sequence; 'exotic species; flocalizaçấo geografica descrita como Northeast littoral of Brazil; 'sPatinga hybrid, which is the result of interspecific hybridisation between a female

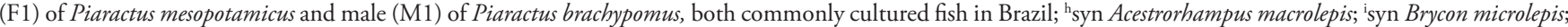
'syn Prochilodus scrofa; 'syn Paulicea luetkeni. 
The biogeographical study of fish species presents in the Brazilian ichthyofauna that are reported as intermediate hosts of Contracaecum larvae are presented in a retrospective study obtained in articles published in Brazil between 1928 and 2018. This study identified 16 Orders (Cichliformes, Perciformes, Characiformes, Siluriformes, Tetraodontiformes, Carcharhiniformes, Clupeiformes, Scorpaeniformes, Gymnotiformes, Gadiformes, Lophiiformes, Ophidiiformes, Pleuronectiformes, Rajiformes, Hexanchiformes, Squatiniformes); 49 families; 96 genera, 140 species and morphotypeg "Patinga" hybrid, which is the result of interspecific hybridisation between a female (F1) of Piaractus mesopotamicus and male (M1) of Piaractus brachypomus, all distributed in three aquatic habitats (Fig. 4). Freshwater fish was the most prevalent, with $56 \%$ of the fish of this environment parasitized by Contracaecum larvae, followed by marine fish (25\%) and estuarine fish (19\%).

The fish of the order Perciformes were the most representative in number of families, while the order Characiformes showed the greatest richness of species that are intermediate hosts of Contracaecum larvae. The order Cichliformes although it showed low diversity in providing hosts, demonstrated its importance by including genera of great economic value to the Amazon region such as Astronotus, Cichla, Crenicichla and Geophagus. In relation to the diversity of hosts and available information, the State of Rio de Janeiro appears first in the number of fishes recorded as having larvae of Anisakidae, followed respectively by the states of Paraná and São Paulo; additionally, these larvae are distributed in another 12 Brazilians states. Among the species with the highest number of scientific records of parasitism by larvae of Contracaecum the piscivorous species, Hoplias malabaricus is the one most cited, besides the species Geophagus brasiliensis and A. ocellatus, the species used in this study, with the mesentery and intestinal serosa being the main sites of infection of this parasite with $52 \%$ and $27 \%$ respectively (Table 1 and 2 ).
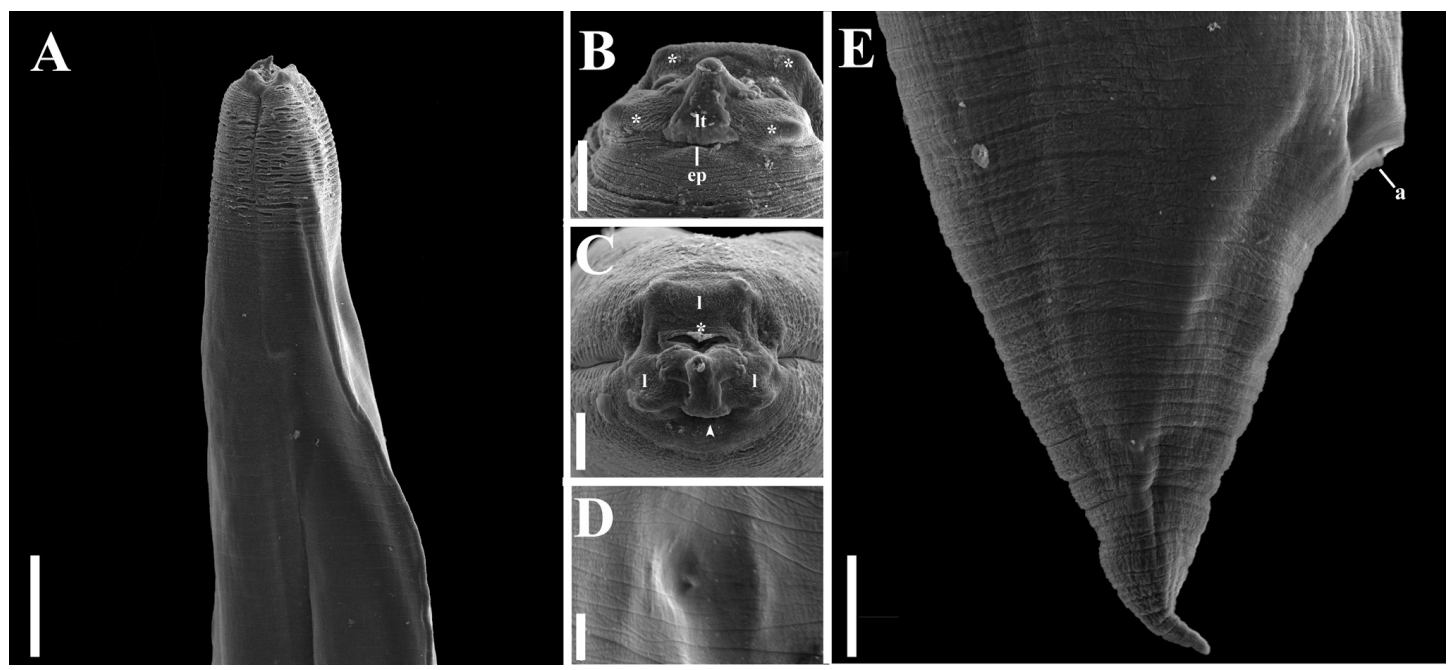

Figure 3. Scanning electron micrographs of Contracaecum sp. (L3 larvae) parasite of $A$. ocellatus: (A) Cephalic region showing evidence cuticle with transversal striations and larval tooth. Bar $=20 \mu \mathrm{m}$; (B) Side view of cephalic region with papillae $\left({ }^{*}\right)$, larval tooth $(\mathrm{lt})$ and excretory pore (ep). Bar $=20 \mu \mathrm{m}$. (C) Frontal view of the oral opening with three lips (l), mouth opening, larval tooth and excretory pore (arrow head). (D) Detail of deirid. Bar $=20 \mu \mathrm{m}$. (E) Posterior portion, (a) anus, the tail without mucron. Bar $=20 \mu \mathrm{m}$.

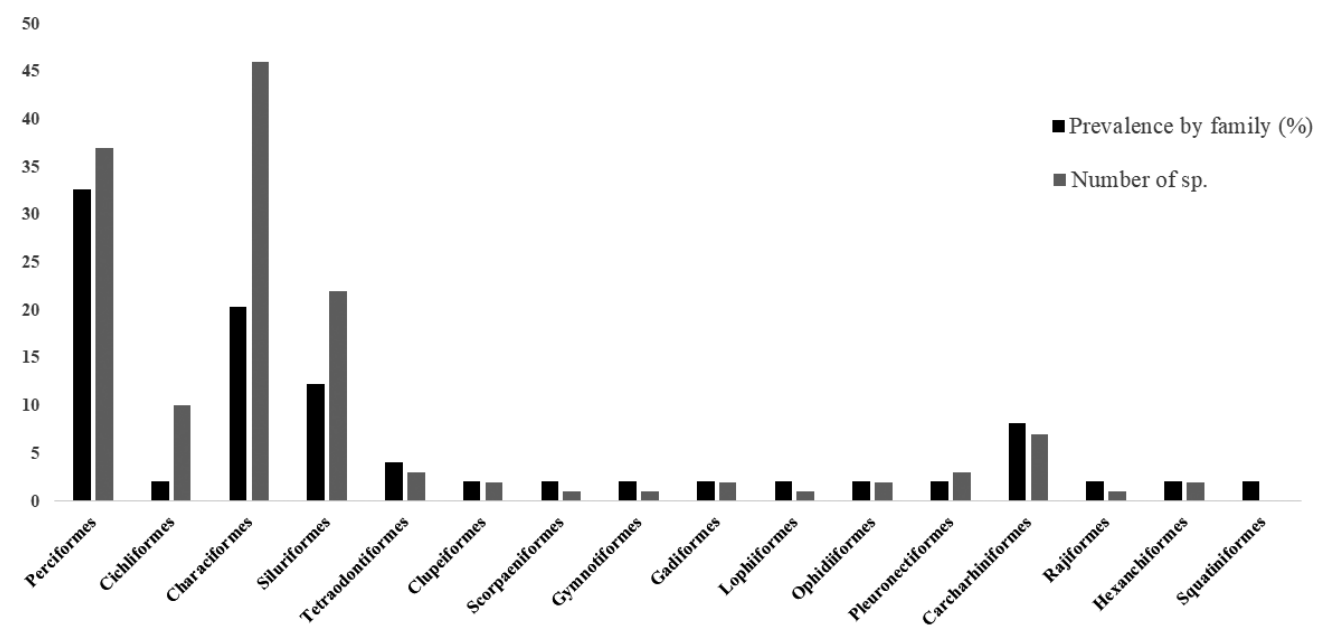

Figure 4. Distribution and prevalence of families by orders and diversity of fish that are hosts of Contracaecum larvae in Brazil. 
Table 2. Morphological and morphometric comparison of third-stage larvae of Contracaecum sp. collected from Astronotus ocellatus commercialized in the state of Pará.

\begin{tabular}{|c|c|c|c|c|c|c|c|}
\hline \multirow{3}{*}{$\begin{array}{c}\text { Character } \\
\text { Hosts larvae }\end{array}$} & \multicolumn{7}{|c|}{ Third stage larvae the Contracaecum spp. } \\
\hline & \multirow{2}{*}{$\begin{array}{l}\text { Astronotus } \\
\text { ocellatus }\end{array}$} & \multicolumn{2}{|l|}{ Type 1} & \multicolumn{2}{|c|}{ Type 2} & \multirow{2}{*}{$\begin{array}{l}\text { Hoplias malabaricus; } \\
\text { Hoplerythinus unitaeniatus }\end{array}$} & \multirow{2}{*}{$\begin{array}{l}\text { Paralichthys } \\
\text { isosceles }\end{array}$} \\
\hline & & \multicolumn{2}{|c|}{$\begin{array}{l}\text { Galeocharax bumeralis; } \\
\text { Rhaphiodon vulpinus }\end{array}$} & \multicolumn{2}{|c|}{$\begin{array}{l}\text { Rhaphiodon vulpinus; } \\
\text { Prochilodus lineatus } \\
\end{array}$} & & \\
\hline Locality & $\begin{array}{l}\text { Santarém- } \\
\text { Pará }\end{array}$ & \multicolumn{2}{|l|}{ Paraná } & & Paraná & Maranháo & Rio de Janeiro \\
\hline Environment & Freshwater & \multicolumn{2}{|c|}{ Freshwater } & & Freshwater & Freshwater & Marine \\
\hline Prevalence & $67.5 \%$ & \multicolumn{2}{|l|}{-} & & - & $100 \%$ and $80 \%$ & $3.3 \%$ \\
\hline Length $^{\mathrm{a}}$ & $11-16$ & \multicolumn{2}{|c|}{$3.89-4.80$} & & $15.70-25.70$ & $12-34.50$ & $4.88-5.06$ \\
\hline Width & $367-533$ & \multicolumn{2}{|c|}{$150-313$} & & $449-843$ & $320-710$ & $90-220^{\mathrm{d}}$ \\
\hline Nervous ring & $260-313$ & \multicolumn{2}{|c|}{$205-225$} & & $313-381$ & $180-370$ & $140-180$ \\
\hline Esophagus ${ }^{\mathrm{L}, \mathrm{ab}}$ & $1-2$ & \multicolumn{2}{|c|}{$537-693$} & & $1.97-2.11$ & $1.87-2.61$ & $640-700$ \\
\hline Esophagus $^{\mathrm{W}}$ & 66-133 & \multicolumn{2}{|l|}{-} & & - & $60-120$ & $30-90$ \\
\hline Intestinal cecum ${ }^{\mathrm{L}, \mathrm{ab}}$ & $1-1.5$ & \multicolumn{2}{|c|}{$310-476$} & & $1.50-1.58$ & $1.20-2.15$ & $280-330$ \\
\hline Intestinal cecum $^{\mathrm{W}, \mathrm{b}}$ & $120-167$ & \multicolumn{2}{|c|}{-} & & - & $160-280$ & - \\
\hline Ventriculus $^{\mathrm{L}, \mathrm{b}}$ & $66-120$ & \multicolumn{2}{|l|}{$24-33$} & & 63 & $60-120$ & - \\
\hline Ventriculus $^{\mathrm{W}, \mathrm{b}}$ & $60-120$ & \multicolumn{2}{|l|}{$33-36$} & & 81 & $60-120$ & $40-100$ \\
\hline $\begin{array}{l}\text { Ventricular } \\
\text { appendix }{ }^{\mathrm{L}, \mathrm{b}}\end{array}$ & $233-460$ & \multicolumn{2}{|c|}{$510-721$} & & $462-503$ & $400-720$ & $420-500$ \\
\hline $\begin{array}{l}\text { Ventricular } \\
\text { appendix }\end{array}$ & $66-93$ & \multicolumn{2}{|l|}{-} & & - & $60-150$ & - \\
\hline Genital primordium $^{a}$ & $3-5$ & \multicolumn{2}{|l|}{-} & & - & - & - \\
\hline Rectum & $110-233$ & - & & & - & - & - \\
\hline Tail & $100-200$ & $78-126$ & & & 95 & $100-220$ & $140-170$ \\
\hline Mucron & Absent & - & & & - & Absent & Absent \\
\hline Reference & $\begin{array}{l}\text { From this } \\
\text { study }\end{array}$ & Moravec et al. & (1993) & Mora & avec et al. (1993) & Martins et al. (2005) & $\begin{array}{c}\text { Felizardo et al. } \\
(2009)\end{array}$ \\
\hline Character & & & Thirc & stage la & Irvae the Contraca & cum spp. & \\
\hline Hosts larvae & $\begin{array}{c}\text { Lophius } \\
\text { gastrophysus }\end{array}$ & $\begin{array}{l}\text { Cynoscion } \\
\text { guatucupa }\end{array}$ & $\begin{array}{r}\text { Pygoc } \\
\text { natt }\end{array}$ & $\begin{array}{l}\text { ntrus } \\
\text { reri }\end{array}$ & $\begin{array}{l}\text { Paralichthys } \\
\text { patagonicus }\end{array}$ & $\begin{array}{c}\text { Xystreurys } \\
\text { rasile }\end{array}$ & $\begin{array}{l}\text { Astyanax } \\
\text { fasciatus }\end{array}$ \\
\hline Type locality & $\begin{array}{l}\text { Rio de } \\
\text { Janeiro }\end{array}$ & Rio de Janeiro & 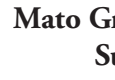 & ssso do & Rio de Janeiro & Rio de Janeiro & Minas Gerais \\
\hline Environment & Marine & Marine & Fresh & jater & Marine & Marine & Freshwater \\
\hline Prevalence & $8.3 \%$ & $6.6 \%$ & 61.8 & & $8.3 \%$ & $13.3 \%$ & $58.11 \%$ \\
\hline Length $^{a}$ & 4.14 & $2.9-4.25$ & $15.11-2$ & 7.25 & $3.05-4.80$ & $3.25-4.30$ & $2.75-4.55$ \\
\hline Width & 90 & $110-150$ & $617-7$ & & $140-190$ & $140-160$ & $93-197$ \\
\hline Nervous ring & 60 & $170-180$ & $160-2$ & & $160-170$ & $160-170$ & $144-220$ \\
\hline Esophagus $^{\mathrm{L}, \mathrm{ab}}$ & 200 & $430-550$ & $1.7-2$ & & $600-650$ & $550-600$ & 201-309 \\
\hline Esophagus ${ }^{\mathrm{W}, \mathrm{b}}$ & - & - & - & & $250-280$ & $250-260$ & $15-42$ \\
\hline Intestinal cecum ${ }^{\mathrm{L}, \mathrm{ab}}$ & 100 & $200-310$ & $1.42-2$ & & $260-300$ & $270-290$ & $227-482$ \\
\hline Intestinal cecum ${ }^{\mathrm{W}, \mathrm{b}}$ & - & - & - & & - & - & \\
\hline Ventriculus $^{\mathrm{L}, \mathrm{b}}$ & 20 & $50-75$ & $114-2$ & & $20-40$ & $40-70$ & $41-83$ \\
\hline Ventriculus $^{\mathrm{W}, \mathrm{b}}$ & 30 & $30-65$ & $76-$ & & $30-35$ & $30-40$ & $11-32$ \\
\hline $\begin{array}{l}\text { Ventricular } \\
\text { appendix }{ }^{\mathrm{L}, \mathrm{b}}\end{array}$ & 360 & $400-470$ & $400-7$ & & $440-460$ & $440-450$ & $367-682$ \\
\hline $\begin{array}{l}\text { Ventricular } \\
\text { appendix }^{\mathrm{W}, \mathrm{b}}\end{array}$ & - & - & - & & $130-160$ & $130-150$ & - \\
\hline Genital primordium $^{a}$ & - & - & - & & - & - & - \\
\hline Rectum & - & - & - & & - & - & - \\
\hline Tail & 120 & $80-110$ & $140-2$ & & $110-140$ & $110-130$ & $74-130$ \\
\hline Mucron & - & Absent & Abs & & Absent & Absnet & Absent \\
\hline
\end{tabular}

Saad et al. Fontenelle et al. Vicentin et al. Fonseca et al. Fonseca et al. Vieira-Menezes et al.

$\begin{array}{lllll}(2012) & \text { (2013) (2013) } \quad \text { (2016) }\end{array}$

${ }^{\mathrm{a}}$ Measurements in micrometers unless indicated; The parameter number of buds is given in amplitude; ${ }^{\mathrm{b}} \mathrm{Abbreviations:} \mathrm{L}=$ length, $\mathrm{W}=\mathrm{width}$; ${ }^{\mathrm{c}} \mathrm{Other}$ hosts cited by Moravec, Kohn, Fernandes 1993: Pseudoplatystoma corruscans; Galeocharax humeralis; Rhaphiodon vulpinus; Hoplias malabaricus; Plagioscion squamosissimus; Crenicichla lepidota; ${ }^{\mathrm{d}}$ Measurement of the width withdrawn in the middle of the body. 


\section{Discussion}

The nematode found parasitizing the intestine and mesentery of $A$. ocellatus of the municipality of Santarém, state of Pará has characteristics similar to the Contracaecum genus, including an oesophagus with small ventricle, ventricular appendix, caecum that extends anteriorly to the nerve ring, an excretory pore opening at the head end slightly posterior to the larval tooth at the base of the ventral lip, oval and transversal buccal opening, surrounded by three lips. According to Moravec (1998), Timi et al. (2001), Felizardo et al. (2009) and Fonseca et al. (2016) these are important characteristics for diagnosing nematodes in the Anisakidae (Skrjabin \& Karokhin, 1945) included Contracaecum (Railliet \& Henry, 1912).

The Contracaecum larvae parasites of $A$. ocellatus present morphological and morphometric similarities with the other described larvae parasitizing different hosts in Brazil; however, we do not consider it appropriate to morphologically and morphometrically the classify larvae found in this work as morphotype I, II as suggested by Moravec et al. (1993). We corroborate Moravec (1998), Moravec et al. (2016) in affirming that the systematics of Contracaecum fish parasites have been based on the morphology of the adult; while systematics of the larvae remains undeveloped, which makes it impossible to attribute more specific taxonomic levels to the larval forms. Morphological and morphometric data on the third-stage larvae Contracaecum parasite of $A$. ocellatus of the municipality of Santarém are compared to the morphometric data of larvae harvested from different hosts in Brazil in Table 2.

Although this is the first record of the prevalence, morphology and morphometry of Contracaecum larvae in the municipality of Santarém, state of Pará in A. ocellatus, a fish of ornamental and food importance in the region, other authors such as Moravec (1998), Azevedo et al. (2007; 2010; 2011) and Luque et al. (2011) have already reported the occurrence of this genus parasitizing A. ocellatus introduced in different localities of Brazil.

Astronotus ocellatus from the Tapajós river in Santarém-PA presented a prevalence of $67.5 \%$ of infection, and a total infection intensity of 150 larvae of Contracaecum encysted in the intestinal serosa and mesentery. Azevedo et al. (2007; 2010; 2011) reported the occurrence of Contracaecum larvae parasitizing $A$. ocellatus introduced in the Rio Guandu in the State of Rio de Janeiro, with a prevalence of 2.8\%. Latini \& Petrere (2004) and Azevedo et al. (2007) state that introduction of exotic fish into new habitats may cause changes in the composition of the local fish fauna to the point of altering the ecological flow, modifying reproduction, growth and development in local species, reducing the abundance of young individuals and parental cross-breeding, all leading to a reduction in the genetic and population biodiversity of some species. These factors influence the parasitic microbiota of native fish, introducing parasites previously not found in these habitats.

For the northern region of Brazil, there are few reports of the occurrence of Contracaecum larvae in commercially important fish, especially the Cichlidae family, since the family is very diverse in the Amazonian biome. For $A$. ocellatus in the northern region, only the works of Neves et al. (2013), Tavares-Dias et al. (2014), Bittencourt et al. (2014) and Tavares-Dias \& Neves (2017) have reported the occurrence and prevalence of parasitism by Contracaecum larvae in the states of Amapá and Amazonas, but the prevalence of $A$. ocellatus infection observed in this study stands out when compared to other states of northern Brazil. Table 3 presents the studies that reported the occurrence and prevalence of Contracaecum larvae in the Amazonian biome in the last 10 years.

In this study the level of parasitic infection of $A$. ocellatus (67.5\%) by Contracaecum sp. was high, while other authors reported parasitism by Contracaecum sp. in different hosts in the Brazilian Amazon region. Salgado (2011) showed a prevalence of $17.5 \%$ of Contracaecum sp. in Cichla spp. commercialized in southeastern Pará; Benigno et al. (2012), analysed Hoplerythrinus unitaeniatus (Spix \& Agassiz, 1829), Hoplias malabaricus (Bloch, 1794) and Pygocentrus nattereri Kner, 1858, species consumed on the island of Marajó and reported a prevalence of 34.31\%, 41.35\% and $58.42 \%$ by Contracaecum larvae, in addition to the occurrence of coinfection with other nematodes. Rodrigues et al. (2015) reported Contracaecum larvae in 5 different species of fish from the northeast of Pará, with prevalence of $60 \%$ in the municipality of Colares and 40\% in Vigia municipality, both in the State of Pará.

Of the 26 Brazilian States and the federal district, the occurrence of Contracaecum larvae was recorded in 15 states. Although the highest prevalence of occurrence is in the studies of freshwater fish, the State of Rio de Janeiro appears first in the number of records in the literature for of Anisakidae larvae, primarily in marine fish. Pavanelli et al. (2013), warn about parasite diversity in fish, reporting that less than $25 \%$ of the Brazilian ichthyofauna have been studied in order to learn about their parasitic fauna, with the Amazon region and Paraná basin the important areas in generating of research into parasites of aquatic organisms, while other regions of Brazil remain as a vast field to be explored.

This work contributes new quantitative data on infections by Contracaecum larvae, informing the population of the occurrence of this parasite in 16 orders, 49 family, 96 genera, 140 species and Patinga hybrid of fish, with the ichthyofauna of the freshwater environment being the ones most cited as intermediate hosts for this genus. According to Pavanelli et al. (2015), the genus Contracaecum has a wide geographic distribution and has been observed in wild fish and in culture systems, having been found parasitizing more than 70 species of fish in almost all regions of the country. For Agostinho et al. (2005) in Brazil, the number of fish in continental aquatic ecosystems is still imprecise and difficult to estimate, especially because of the number of unregulated watersheds, infrastructure required for sampling, dispersal information or often difficult to access, and the need for taxonomic revision for several groups.

Hoplias malabaricus, Geophagus brasiliensis and Astronotus ocellatus, the species used in herein are generally carnivorous (FROESE \& PAULY, 2018). H. malabaricus and A. ocellatus are preferentially piscivorous as adults, but feed on plankton, crustaceans, insects and seeds as juveniles (SANTOS et al., 2006; FROESE \& PAULY, 2018). Micro-crustaceans are the first intermediate hosts and fish act as second intermediate hosts or as paratenic hosts of Contracaecum larvae, while piscivorous birds are the definitive hosts (MOREIRA et al., 2009; MORAVEC, 2009).

The species of the orders Perciformes and Characiformes were the most cited as intermediate hosts of Contracaecum larvae. 
Table 3. Prevalence and comparison of larvae of Contracaecum sp. the parasite Astronotus ocellatus is commercialized in the state of Pará and compared to larvae of Contracaecum spp. larvae of parasites of different hosts of northern Brazil registered in the last 10 years.

\begin{tabular}{|c|c|c|c|}
\hline Host & Locality & Prevalence (\%) & Reference \\
\hline \multirow{5}{*}{ Astronotus ocellatus } & Pará & 67.5 & From this study \\
\hline & Amapá & 53 & Neves et al. (2013) \\
\hline & Amazon & 25 & Tavares-Dias et al. (2014) \\
\hline & Amapá & 38.5 & Bittencourt et al. (2014) \\
\hline & Amapá & 24.2 & Tavares-Dias \& Neves (2017) \\
\hline \multirow[t]{4}{*}{ Hoplias malabaricus } & Pará & 43 & Benigno et al. (2012) \\
\hline & Amapá & $51.5 ; 33.3 ; 6.1^{\mathrm{a}}$ & Alcântara \& Tavares-Dias (2015) \\
\hline & Amapá & $69.7 ; 70.67^{\mathrm{b}}$ & Gonçalves et al. (2016) \\
\hline & Amapá & $83.3 ; 10 ; 10 ; 3.3^{\mathrm{c}}$ & Oliveira et al. (2018) \\
\hline \multirow[t]{4}{*}{ Hoplerythrinus unitaeniatus } & Pará & 35 & Benigno et al. (2012) \\
\hline & Amapá & $63.3 ; 20^{\mathrm{d}}$ & Alcântara \& Tavares-Dias (2015) \\
\hline & Amapá & $76.75 ; 74.4^{\mathrm{b}}$ & Gonçalves et al (2016) \\
\hline & Amapá & $90 ; 3.3^{\mathrm{e}}$ & Oliveira et al. (2018) \\
\hline \multirow[t]{2}{*}{ Pygocentrus nattereri } & Pará & 59 & Benigno et al. (2012) \\
\hline & Amapá & 53.3 & Brito-Junior \& Tavares-Dias (2018) \\
\hline Metynnis lippincottianus & Amapá & 68.7 & Hoshino and Tavares-Dias (2014) \\
\hline Hemibrycon surinamensis & Amapá & 15.1 & Hoshino et al. (2014) \\
\hline Plagioscion squamosissimus & Pará & 70 & Rodrigues et al. (2015) \\
\hline Brachyplatystoma filamentosum & Pará & 68.18 & Rodrigues et al. (2015) \\
\hline Brachyplatystoma rousseauxii & Pará & 90 & Rodrigues et al. (2015) \\
\hline Oxydoras niger & Pará & 10 & Rodrigues et al. (2015) \\
\hline Metynnis hypsauchen & Amapá & 3.3 & Oliveira et al. (2015) \\
\hline Piaractus brachypomus & Amapá & $32.3 ; 14.7 ; 5.9^{f}$ & Oliveira \& Tavares-Dias (2016) \\
\hline Acestrorhynchus falcatus & Amapá & 93.4 & Hoshino et al. (2016) \\
\hline Acestrorhynchus falcirostris & Amapá & 81.8 & Hoshino et al. (2016) \\
\hline Triportheus angulatus & Amapá & 3.3 & Oliveira et al. (2016) \\
\hline Trachelyopterus coriaceus & Amapá & 17.1 & Pantoja et al. (2016) \\
\hline Trachelyopterus galeatus & Amapá & 13.5 & Pantoja et al. (2016) \\
\hline Brycon amazonicus & Amazonas & $10 ; 16.13^{\mathrm{g}}$ & Ribeiro et al. (2016) \\
\hline Brycon melanopterus & Amazonas & 60 & Ribeiro et al. (2016) \\
\hline Cicbla monoculus & Amazonas & 7.89 & Santana et al. (2017) \\
\hline Gymnotus carapo & Amapá & 60 & Brito-Junior \& Tavares-Dias (2018) \\
\hline Astyanax abramis & Amapá & 33.3 & Brito-Junior \& Tavares-Dias (2018) \\
\hline Astyanax sp. & Amapá & 40 & Brito-Junior \& Tavares-Dias (2018) \\
\hline
\end{tabular}

${ }^{a}$ Prevalence by site of infection intestine, caecum and liver respectively; ${ }^{b}$ Seasonal variation of parasitism prevalence according to the dry season and rainy season

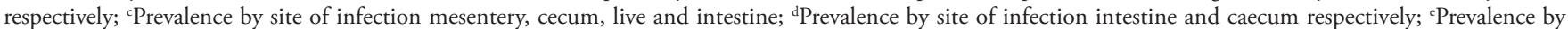

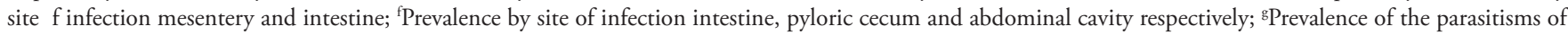
Contracaecum sp. of Brycon amazonicus collected in Rio Negro and Solimóes respectively.

Takemoto \& Lizama (2010) noted the low host specificity for nematodes, especially in the larval stage. Parasitism in these two orders, in addition to the order Cichliformes, is important because they harbor that are widely consumed in the northern region, such as apaiari (A. ocellatus), tucunaré (Cichla spp.), pescada branca (Plagioscion squamosissimus), pescada (Macrodon ancylodon), lambarí (Astyanax spp.), Piau (Leporinus spp.), as well as fish of high importance for the Brazilian aquaculture sector, such as pacu (Piaractus mesopotamicus), pirapitinga (Piaractus brachypomus), matrinxã (Brycon spp.), Curimbata (Prochilodus spp.), and other species, which are commercialized as ornamental fish or are important elements in the ecological flow of Amazonian aquatic environments, even though they are not consumed by the Brazilian population, especially in the northern region.

\section{Conclusions}

The morphological, morphometric, biogeographic and prevalence data of the Contracaecum larvae provided here are important because they reinforce $A$. ocellatus as the intermediate host of this nematode. In addition to the aquariophilic value of A. ocellatus that is important to the Amazonian region as a source of foreign exchange, this fish is part of the food base for riverside populations, and is also consumed by other animals that live on the 
banks of the Tapajós river, and are therefore potential intermediate or definitive hosts of this parasite, either by completing the cycle or by increasing its geographical distribution.

\section{Acknowledgements}

The authors are grateful to the following: the Laboratório de Histologia e Embriologia Animal and Laboratório de Microscópia Eletrônica de Varredura - Instituto da Saúde e Produção Animal - Universidade Federal Rural da Amazônia - UFRA, campus Belém, state of Pará, Brazil for the use of the scanning electron microscope. This study is part of the Ph.D. thesis of Raul Henrique da Silva Pinheiro, developed for the Programa de Pós-Graduação em Biologia de Agentes Infecciosos e Parasitários, Instituto de Ciências Biológicas, Universidade Federal do Pará-UFPA. This study was financed in part by the Coordenação de Aperfeiçoamento de Pessoal de Nível Superior - Brasil (CAPES) - Finance Code 001, PAPQ2018/PROPESP/UFPA and Ministério da Educação do Brasil.

\section{References}

Abdallah VD, Azevedo RK, Carvalho ED, Silva RJ. New hosts and distribution records for nematode parasites of freshwater fishes from São Paulo State, Brazil. Neotrop Helminthol 2012; 6(1): 43-57.

Agostinho AA, Thomaz SM, Gomes LC. Conservação da biodiversidade em águas continentais do Brasil. Megadiversidade 2005; 1(1): 70-78.

Alarcos AJ, Pereira AN, Taborda NL, Luque JL, Timi JT. Parasitological evidence of stocks of Paralichthys isosceles (Pleuronectiformes: Paralichthyidae) at small and large geographical scales in South American Atlantic coasts. Fish Res 2016; 173(3): 221-228. http://dx.doi.org/10.1016/j. fishres.2015.07.018.

Alcântara NM, Tavares-Dias M. Structure of the parasites communities in two Erythrinidae fish from Amazon river system (Brazil). Rev Bras Parasitol Vet 2015; 24(2): 183-190. http://dx.doi.org/10.1590/S198429612015039. PMid:26083690.

Alves DR, Luque JL, Abdallah VD. Metazoan parasites of chub mackerel, Scomber japonicus (Osteichthyes: Scombridae), from the coastal zone of the State of Rio de Janeiro, Brazil. Rev Bras Parasitol Vet 2003; 12(4): 164-170.

Alves DR, Luque JL, Paraguassú AR. Community ecology of the metazoan parasites of pink cusk-eel, Genypterus brasiliensis (Osteichthyes: Ophidiidae), from the coastal zone of the State of Rio de Janeiro, Brazil. Mem Inst Oswaldo Cruz 2002; 97(5): 683-689. http://dx.doi.org/10.1590/ S0074-02762002000500018. PMid:12219136.

Alves DR, Luque JL. Community ecology of the metazoan parasites of white croaker, Micropogonias furnieri (Osteichthyes: Sciaenidae), from the coastal zone of the State of Rio de Janeiro, Brazil. Mem Inst Oswaldo Cruz 2001a; 96(2): 145-153. http://dx.doi.org/10.1590/S007402762001000200002. PMid:11285488.

Alves DR, Luque JL. Community ecology of the metazoan parasites of five Scombrid species (Perciforme: Scombridae), from the coastal zone of the State of Rio de Janeiro, Brasil. Rev Bras Parasitol Vet 2006; 15(4): 167-181. PMid:17196121.

Alves DR, Luque JL. Quantitative aspects of metazoan parasite infrapopulations of Micropogonias furnieri (Osteichthyes: Sciaenidae) from the coastal zone of the state of Rio de Janeiro, Brazil. Parasitol Día 2001b; 25(1-2): 30-35.

Alves DR, Paraguassú AR, Luque JL. Community ecology of the metazoan parasites of the grey triggerfish, Balistes capriscus Gmelin, 1789 and queen triggerfish $B$. vetula Linnaeus, 1758 (Osteichthyes: Balistidae) from the state of Rio de Janeiro, Brazil. Rev Bras Parasitol Vet 2005; 14(2): 71-77. PMid:16153348.

Alves DR, Paraguassú AR, Luque JL. Metazoan parasites of the Brazilian codling, Urophycis brasiliensis (Kaup, 1858) (Osteichthyes: Phycidae) from the coastal zone of the State of Rio de Janeiro, Brazil. Rev Bras Parasitol Vet 2004; 13(1): 49-55.

Azevedo RK, Abdallah VD, Luque JL. Acanthocephala, Annelida, Arthropoda, Myxozoa, Nematoda and Platyhelminthes parasites of fishes from the Guandu River, Rio de Janeiro, Brazil. Check List 2010; 6(4): 659-667. http://dx.doi.org/10.15560/6.4.659.

Azevedo RK, Abdallah VD, Luque JL. Biodiversity of fish parasites from Guandu river, Southeastern Brazil: an ecological approach. Neotrop Helminthol 2011; 5(2): 185-199.

Azevedo RK, Abdallah VD, Luque JL. Community ecology of metazoan parasites of apaiarí Astronotus ocellatus (Cope, 1872) (Perciformes: Cichlidae) from Guandu river, State of Rio de Janeiro, Brazil. Rev Bras Parasitol Vet 2007; 16(1): 15-20. PMid:17588317.

Azevedo RK, Abdallah VD, Luque JL. Ecologia da comunidade de metazoários parasitos do acará Geophagus brasiliensis (Quoy e Gaimard, 1824) (Perciformes: Cichlidae) do rio Guandu, Estado do Rio de Janeiro, Brasil. Acta Sci Biol Sci 2006; 28(4): 403-411.

Barros GC, Amato JFR. Larvas de anisakídeos de peixe-espada, Trichiurus lepturus L., da costa do Estado do Rio de Janeiro, Brasil. Rev Bras Biol 1993; 53(2): 241-245.

Barros GC, Cavalcanti JW. Infected larvae belonging to the groups of Anisaksis from Northeast Coast - Brazil. Hig Aliment 1998; 12(58): 71-75.

Barros GC. Larvas de anisakídeos de peixes economicamente importantes da costa do Rio de Janeiro. Rev Bras Med Vet 1994; 16(5): 205-208.

Barros LA, Mateus LAF, Braum DT, Bonaldo J. Ecological aspects of endoparasites in red piranha (Pygocentrus nattereri Kner, 1860) from Cuiabá river, Mato Grosso, Brazil. Arq Bras Med Vet Zootec 2010; 62(1): 228-231. http://dx.doi.org/10.1590/S0102-09352010000100033.

Barros LA, Moraes J Fo, Oliveira RL. Nematode larvae with zoonotical importance found in trairas (Hoplias malabaricus) in Santo Antonio do Leverger, Mato Grosso, Brazil. Arq Bras Med Vet Zootec 2007; 59(2): 533-535. http://dx.doi.org/10.1590/S0102-09352007000200042.

Barros LA, Moraes J Fo, Oliveira RL. Nematodes with zoonotical potencial in fishes with economical importance from Cuiabá river. $R$ Bras $C i$ Vet 2006; 13(1): 55-57.

Barros LA, Oliveira RL, Moraes J Fo, Justino CHS, Mateus LAF. Analisys of the parasitism by Contracaecum sp. and Eustrongylides sp. in cacharas, Pseudoplatystoma fasciatum (Linnaeus, 1766) (Pisces: Pimelodidae) from Cuiabá river, Mato Grosso. R Bras Ci Vet 2009; 16(2): 58-61.

Barros LA, Tortelly R, Pinto RM, Gomes DC. Effects of experimental infections with larvae of Eustrongylides ignotus Jäegerskiold, 1909 and Contracaecum multipapillatum (Drasche, 1882) Baylis, 1920 in rabbits. Arq Bras Med Vet Zootec 2004; 56(3): 325-332. http://dx.doi.org/10.1590/ S0102-09352004000300007.

Bautista-Hernández CE, Monks S, Pulido-Flores G, Rodríguez-Ibarra AE. Revisión bibliográfica de algunos términos ecológicos usados en 
parasitología, y su aplicación en estudios de caso. In: Pulido-Flores G, Monks S, López-Herrera M. Estudios en biodiversidad. vol. I. Lincoln, Nebraska: Zea Books; 2015. p. 11-19. (Zea e-books; book 35).

Bellay S, Ueda BH, Takemoto RM, Lizama MAP, Pavanelli GC. Fauna parasitária de Geophagus brasiliensis (Perciformes: Cichlidae) em reservatórios do estado do Paraná, Brasil. R Bras Bioci 2012; 10(1): 74-78.

Benigno RNM, São Clemente SC, Matos ER, Pinto RM, Gomes DC, Knoff M. Nematodes in Hoplerytrinus unitaeniatus, Hoplias malabaricus and Pygocentrus nattereri (Pisces: Characiformes) in Marajó Island, Brazil. Rev Bras Parasitol Vet 2012; 21(2): 165-170. http://dx.doi.org/10.1590/ S1984-29612012000200018. PMid:22832760.

Bittencourt LS, Pinheiro DA, Cárdenas MQ, Fernandes BM, Tavares-Dias M. Parasites of native Cichlidae populations and invasive Oreochromis niloticus (Linnaeus, 1758) in tributary of Amazonas River (Brazil). Rev Bras Parasitol Vet 2014; 23(1): 44-54. http://dx.doi.org/10.1590/S198429612014006 . PMid:24728360.

Brasil. Classificação de risco dos agentes biológicos. 2. ed. Brasília, Ministério da Saúde; 2010. (Normas e manuais técnicos; série 309A).

Brito-Junior IA, Tavares-Dias M. Metazoários parasitos de quatro espécies de peixes da bacia Igarapé Fortaleza, estado do Amapá (Brasil). Biota Amaz 2018; 8(2): 1-3.

Bush AO, Lafferty KD, Lotz JM, Shostak AW. Parasitology meets ecology on its own terms: Margolis et al. revisited. J Parasitol 1997; 83(4): 575583. http://dx.doi.org/10.2307/3284227. PMid:9267395.

Camargo AA, Leite LAR, Azevedo RK, Abdallah VD. Parasites of Astyanax bockmanni (Characiformes, Characidae) collected from the Batalha River, Sáo Paulo State, Brazil. Environmental 2016b; 1(1): 56-59.

Camargo AA, Negrelli DC, Pedro NHO, Azevedo RK, Silva RJ, Abdallah VD. Metazoan parasite of lambari Astyanax altiparanae, collected from the Peixe river, São Paulo, southeast of Brazil. Cienc Rural 2016a; 46(5): 876-880. http://dx.doi.org/10.1590/0103-8478cr20151100.

Camargo AA, Pedro NHO, Pelegrini LS, Azevedo RK, Silva RJ, Abdallah VD. Parasites of Acestrorhynchus lacustris (Lütken, 1875) (Characiformes: Acestrorhynchidae) collected from the Peixe River, southeast Brazil. Acta Sci Biol Sci 2015; 37(2): 231-237. http://dx.doi.org/10.4025/ actascibiolsci.v37i2.24303.

Campos CM, Fonseca VE, Takemoto RM, Moraes FR. Fauna parasitária de cachara Pseudoplatystoma fasciatum (Siluriforme: Pimelodidae) do rio Aquidauana, Pantanal Sul Mato-grossense, Brasil. Acta Sci Biol Sci 2008; 30(1): 91-96. http://dx.doi.org/10.4025/actascibiolsci.v30i1.1469.

Carvalho AR, Martins RT, Bellei PM, Lima SS. Aspectos ecológicos da helmintofauna de Hoplias malabaricus (Bloch, 1794) (Characiformes, Erythrinidae) da Represa Dr. João Penido (Juiz de Fora-MG, Brasil). Rev Bras Zoociênc 2017; 18(1): 7-20.

Carvalho AR, Tavares LER, Luque JL. Variação sazonal dos metazoários parasitos de Geophagus brasiliensis (Perciformes: Cichlidae) no rio Guandu, Estado do Rio de Janeiro, Brasil. Acta Sci Biol Sci 2010; 32(2): 159-167.

Carvalho S, Guidelli GM, Takemoto RM, Pavanelli GC. Ecological aspects of endoparasite fauna of Acestrorhynchus lacustris (Lütken, 1875) (Characiformes, Acestrorhynchidae) on the Upper Paraná River floodplain, Brazil. Acta Sci Biol Sci 2003; 25(2): 479-483. http://dx.doi.org/10.4025/ actascibiolsci.v25i2.2043.

Casali GP, Takemoto RM. Endoparasitic fauna of Serrasalmus spp. (Characidae: Serrasalminae) in a neotropical floodplain. Acta Sci Biol Sci 2016; 38(1): 105-112. http://dx.doi.org/10.4025/actascibiolsci. v38i1.28592.
Chaves ND, Luque JL. Ecology of metazoans parasites of Menticirrhus americanus (Osteichthyes: Sciaenidae), coast area from Rio de Janeiro State, Brazil. Rev Bras Parasitol Vet 1999; 8(2): 137-144.

Cordeiro AS, Luque JL. Community ecology of the metazoan parasites of atlantic moonfish, Selene setapinnis (Osteichthyes: Carangidae) from the coastal zone of the state of Rio de Janeiro, Brazil. Braz J Biol 2004; 64(3): 399-406. PMid:15622838.

Cordeiro AS, Luque JL. Metazoários parasitos do coió Dactylopterus volitans (Linnaeus, 1758) (Osteichthyes: Dactylopteridae) do litoral do Estado do Rio de Janeiro, Brasil. Acta Sci Biol Sci 2005; 27(2): 119-123.

Dias FJE, São Clemente SC, Knoff M. Nematoides anisaquídeos e cestoides Trypanorhyncha de importância em saúde pública em Aluterus monoceros (Linnaeus, 1758) no estado do Rio de Janeiro, Brasil. Rev Bras Parasitol Vet 2010; 19(2): 94-97. http://dx.doi.org/10.1590/S198429612010000200005 . PMid:20624345.

Dias FJE, São Clemente SC, Pinto RM, Knoff M. Anisakidae nematodes and Trypanorhyncha cestodes of hygienic importance infecting the king mackerel Scomberomorus cavalla (Osteichthyes: Scombridae) in Brazil. Vet Parasitol 2011; 175(3-4): 351-355. http://dx.doi.org/10.1016/j. vetpar.2010.10.014. PMid:21115292.

Dias JS, Pozza A, Pesenti TC, Pereira J Jr, Berne MEA. Helmintos parasitos de Rhamdia quelen (Quoy \& Gaimard, 1824) no sul do Brasil. Sci Anim Health 2016; 4(1): 2-20.

Domingues SAM, Alves DR. Metazoários parasitos de Thyrsitops lepidopoides (Osteichthyes: Gempylidae) do litoral do Estado do Rio de Janeiro, Brasil. Cad UniFOA 2015; 27: 95-103.

Eiras JC, Pavanelli GC, Takemoto RM, Yamaguchi MU, Karkling LC, Nawa Y. Potential risk of fish-borne nematode infections in humans in Brazil - Current status based on a literature review. Food Waterborne Parasitol 2016; 5: 1-6. http://dx.doi.org/10.1016/j.fawpar.2016.08.002.

Eiras JC, Rego AA. Histopatologia em peixes resultantes de infecçóes parasitárias. Publ Inst Zool Dr Augusto Nobre 1989; 208(1): 1-2.

Eiras JC, Takemoto RM, Pavanelli GC. Diversidade dos parasitas de peixes de água doce do Brasil. Maringá: Cliche Tec Editora; 2010.

Fábio SP. Sobre alguns nematodas parasitos de Hoplias malabaricus. Arq Univ Fed Rur Rio de J 1982; 5(2): 179-186.

Felizardo NN, Knoff M, Pinto RM, Gomes DC. Larval anisakid nematodes of the flounder, Paralichthys isosceles Jordan, 1890 (Pisces: Teleostei) from Brazil. Neotrop Helminthol 2009; 3(2): 57-64.

Ferreira DO, Tavares-Dias M. Ectoparasites and endoparasites community of Ageneiosus ucayalensis (Siluriformes: Auchenipteridae), catfish from Amazon River system in northern Brazil. J Parasit Dis 2017; 41(3): 639646. http://dx.doi.org/10.1007/s12639-016-0857-3. PMid:28848252.

Firouzbakhsh F, Noori F, Khalesi MK, Jani-Khalili K. Effects of a probiotic, protexin, on the growth performance and hematological parameters in the Oscar (Astronotus ocellatus) fingerlings. Fish Physiol Biochem 2011; 37(4): 833-842. http://dx.doi.org/10.1007/s10695-011-9481-4. PMid:21487694.

Fonseca MCG, Knoff M, Felizardo NN, Di Azevedo MIN, Torres EJL, Gomes DC, et al. Integrative taxonomy of Anisakidae and Raphidascarididae (Nematoda) in Paralichthys patagonicus and Xystreurys rasile (Pisces: Teleostei) from Brazil. Int J Food Microbiol 2016; 235(17): 113-124. http://dx.doi.org/10.1016/j.ijfoodmicro.2016.07.026. PMid:27491056.

Fontenele O, Nepomuceno FH. Exame dos resultados da introdução do Astronotus ocellatus (Agassiz, 1849), em açudes do Nordeste do Brasil. Bol Téc DNOCS 1983; 41(1): 85-99. 
Fontenelle G, Knoff M, Felizardo NN, Lopes LMS, São Clemente SC. Nematodes of zoonotic importance in Cynoscion guatucupa (Pisces) in the state of Rio de Janeiro. Rev Bras Parasitol Vet 2013; 22(2): 281-284. http://dx.doi.org/10.1590/S1984-29612013005000019. PMid:23778824.

Franceschini L, Zago AC, Schalch SHC, Garcia F, Romera DM, Silva RJ. Parasitic infections of Piaractus mesopotamicus and hybrid (P. mesopotamicus $\mathrm{x}$ Piaractus brachypomus) cultured in Brazil. Rev Bras Parasitol Vet 2013a; 22(3): 407-414. http://dx.doi.org/10.1590/S1984-29612013000300015. PMid:24142174.

Franceschini L, Zago AC, Zocoller-Seno MC, Veríssimo-Silveira R, Ninhaus-Silveira A, Silva RJ. Endohelminths in Cichla piquiti (Perciformes, Cichlidae) from the Paraná River, São Paulo State, Brazil. Rev Bras Parasitol Vet 2013b; 22(4): 475-484. http://dx.doi.org/10.1590/S198429612013000400006. PMid:24473871.

Froese R, Pauly D, editors. FishBase [online]. Mumbai: FishBase Team; 2018 [cited 2018 Jun 18]. Available from: www.fishbase.org

Gonçalves RA, Oliveira MSB, Neves LR, Tavares-Dias M. Seasonal pattern in parasite infracommunities of Hoplerythrinus unitaeniatus and Hoplias malabaricus (Actinopterygii: Erythrinidae) from the Brazilian Amazon. Acta Parasitol 2016; 61(1): 119-129. http://dx.doi.org/10.1515/ ap-2016-0016. PMid:26751882.

Graça RJ, Machado MH. Ocorrência e aspectos ecológicos de metazoários parasitos de peixes do Lago do Parque do Ingá, Maringá, Estado do Paraná. Acta Sci Biol Sci 2007; 29(3): 321-326. http://dx.doi.org/10.4025/ actascibiolsci.v29i3.507.

Guidelli G, Tavechio WLG, Takemoto RM, Pavanelli GC. Fauna parasitária de Leporinus lacustris e Leporinus friderici (Characiformes, Anostomidae) da planície de inundação do alto rio Paraná, Brasil. Acta Sci Biol Sci 2006; 28(3): 281-290.

Guidelli G, Tavechio WLG, Takemoto RM, Pavanelli GC. Relative condition factor and parasitism in anostomid fishes from the floodplain of the Upper Paraná River, Brazil. Vet Parasitol 2011; 177(1-2): 145-151. http://dx.doi.org/10.1016/j.vetpar.2010.11.035. PMid:21176864.

Guidelli GM, Isaac A, Takemoto RM, Pavanelli GC. Endoparasite infracommunities of Hemisorubim platyrhynchos (Valenciennes, 1840) (Pisces: Pimelodidae) of the baía river, upper Paraná River floodplain, Brazil: specific composition and ecological aspects. Braz J Biol 2003; 63(2): 261-268. PMid:14509848.

Guimarães JF, Cristofaro R. Contribuição ao estudo da fauna helmintológica de peixes do Estado da Bahia. Atas Soc Biol 1974; 17(1): 81-85.

Hoshino MDFG, Hoshino EM, Tavares-Dias M. First study on parasites of Hemibrycon surinamensis (Characidae), a host from the eastern Amazon region. Rev Bras Parasitol Vet 2014; 23(3): 343-347. http://dx.doi. org/10.1590/S1984-29612014069. PMid:25271454.

Hoshino MDFG, Neves LR, Tavares-Dias M. Parasite communities of the predatory fish, Acestrorhynchus falcatus and Acestrorhynchus falcirostris, living in sympatry in Brazilian Amazon. Rev Bras Parasitol Vet 2016; 25(2): 207-216. http://dx.doi.org/10.1590/S1984-29612016038. PMid:27334822.

Hoshino MDFG, Tavares-Dias M. Ecology of parasites of Metynnis lippincottianus (Characiformes: Serrasalmidae) from the eastern Amazon region, Macapá, State of Amapá, Brazil. Acta Sci Biol Sci 2014; 36(2): 249-255. http://dx.doi.org/10.4025/actascibiolsci.v36i2.19876.

Isaac A, Guidelli GM, França JG, Pavanelli GC. Composição e estrutura das infracomunidades endoparasitárias de Gymnotus spp. (Pisces: Gymnotidae) do rio Baía, Mato Grosso do Sul, Brasil. Acta Sci Biol Sci 2004; 26(4): 453-462. http://dx.doi.org/10.4025/actascibiolsci.v26i4.1527.

Justino CHS, Barros LA. In vitro evaluation of the resistence of the Contracaecum sp. larvae (Railliet \& Henry, 1912) (Nematoda: Anisakidae), to the essential oil of citronella (Cymbopogon sp.) (Poaceae). $\mathrm{R}$ Bras $\mathrm{C} i$ Vet 2008; 15(3): 122-125.

Karling LC, Lacerda ACF, Alvarenga FMS, Takemoto RM, Pavanelli GC. Endoparasites of Rhaphiodon vulpinus (Characiformes: Cynodontidae) and their relation to the standard length and sex of their hosts. Biosci $J$ 2013b; 29(5): 1311-1316.

Karling LC, Lacerda ACF, Takemoto RM, Pavanelli GC. Ecological relationships between endoparasites and the fish Salminus brasiliensis (Characidae) in a neotropical floodplain. Neotrop Helminthol 2013a; 7(2): 219-230.

Kloss GR. Helmintos parasitos de espécies simpátricas de Astyanax (Pisces, Characidae). Pap Av Dep Zool SP 1966; 18(7): 189-219.

Knoff M, São Clemente SC, Fonseca MCG, Andrada CG, Padovani RES, Gomes DC. Anisakidae parasites of cusk-eel, Genypterus brasiliensis Regan, 1903 purchased in the Rio de Janeiro state, Brazil and of interest in public health. Parasitol Latinoam 2007; 62(3-4): 127-133. http:// dx.doi.org/10.4067/S0717-77122007000200005.

Knoff M, São Clemente SC, Fonseca MCG, Felizardo NN, Lima FC, Pinto RM, et al. Anisakidae nematodes in the blackfin goosefish, Lophius gastrophysus Miranda-Ribeiro, 1915 purchased in the State of Rio de Janeiro, Brazil. Acta Sci Biol Sci 2013; 35(1): 129-133. http://dx.doi. org/10.4025/actascibiolsci.v35i1.12185.

Knoff M, São Clemente SC, Pinto RM, Gomes DC. Nematodes of Elasmobranch fishes from the southern coast of Brazil. Mem Inst Oswaldo Cruz 2001; 96(1): 81-87. http://dx.doi.org/10.1590/S007402762001000100009. PMid:11285478.

Kohn A, Fernandes BMM, Pipolo HV, Godoy MP. Helmintos parasitos de peixes das Usinas Hidrelétricas da Eletrosul (Brasil). II. Reservatórios de Salto Osório e de Salto Santiago, Bacia do Rio Iguaçu. Mem Inst Oswaldo Cruz 1988; 84(3): 299-303. http://dx.doi.org/10.1590/S007402761988000300006 .

Kohn A, Fernandes BMM, Pipolo HV, Godoy MP. List of helminth parasites of fishes from the Passo Fundo Reservoir, Uruguay River basin, Brazil. Mem Inst Oswaldo Cruz 1989; 84(3): 427-428. http://dx.doi. org/10.1590/S0074-02761989000300021.

Lacerda ACF, Santin M, Takemoto RM, Pavanelli GC, Bialetzki A, Tavernari FC. Helminths parasitizing larval fish from Pantanal, Brazil.J Helminthol 2009; 83(1): 51-55. http://dx.doi.org/10.1017/S0022149X08092171. PMid:18854057.

Latini AO, Petrere M Jr. Reduction of a native fish fauna by alien species: an example from brazilian freshwater tropical lakes. Fish Manag Ecol 2004; 11(2): 71-79. http://dx.doi.org/10.1046/j.1365-2400.2003.00372.x.

Leão ELM, Leite RG, Chaves PTC, Ferraz R. Aspectos da reprodução, alimentação e parasitofauna de uma espécie rara de piranha, Serrassalmus altuvei Ramirez, 1965 (Pisces Serrasalmidae) do baixo rio Negro. Rev Bras Biol 1991; 51(3): 545-553.

Leite LAR, Pedro NHO, Azevedo RK, Kinoshita A, Gennari RF, Watanabe S, et al. Contracaecum sp. parasitizing Acestrorhynchus lacustris as a bioindicator for metal pollution in the Batalha River, southeast Brazil. Sci Total Environ 2017; 575(1): 836-840. http://dx.doi.org/10.1016/j. scitotenv.2016.09.132. PMid:27680988. 
Lizama MAP, Takemoto RM, Pavanelli GC. Ecological aspects of metazoan parasites of Astyanax altiparanae Garutti \& Britski, 2000 (Characidae) of the upper Paraná River floodplain, Brazil. Bol Inst Pesca 2008; 34(4): 527-533.

Luque JL, Aguiar JC, Vieira FM, Gibson DI, Santos CP. Checklist of Nematoda associated with the fishes of Brazil. Zootaxa 2011; 3082(1): 1-88.

Luque JL, Alves DR, Ribeiro RS. Community ecology of the metazoan parasites of Banded Croaker, Paralonchurus brasiliensis (Osteichthyes: Sciaenidae), from the coastal zone of the State of Rio de Janeiro, Brazil. Acta Sci Biol Sci 2003; 25(2): 273-278. http://dx.doi.org/10.4025/ actascibiolsci.v25i2.2009.

Luque JL, Chaves ND. Ecologia da comunidade de metazoários parasitos da anchova Pomatomus saltator (Linnaeus) (Osteichthyes, Pomatomidae) do litoral do estado do Rio de Janeiro, Brasil. Rev Bras Zool 1999; 16(3): 711-723. http://dx.doi.org/10.1590/S0101-81751999000300010.

Luque JL, Cordeiro AS, Oliva ME. Metazoan parasites as biological tags for stock discrimination of whitemouth croaker Micropogonias furnieri. J Fish Biol 2010; 76(3): 591-600. http://dx.doi.org/10.1111/j.10958649.2009.02515.x. PMid:20666899.

Luque JL, Porrozzi F, Alves DR. Community ecology of the metazoan parasites of argentine goatfish, Mullus argentinae (Osteichthyes: Mullidae), from the coastal zone of the state of Rio de Janeiro, Brazil. Rev Bras Parasitol Vet 2002; 11(1): 33-38.

Luque JL, Poulin R. Use of fish as intermediate hosts by helminth parasites: a comparative analysis. Acta Parasitol 2004; 49(4): 353-361.

Machado MH, Pavanelli GC, Takemoto RM. Influence of host's sex and size on endoparasitic infrapopulations of Pseudoplatystoma corruscans and Schizodon borelli (Osteichthyes) of the high Paraná river, Brazil. Rev Bras Parasitol Vet 1994; 3(2): 143-148.

Machado MH, Pavanelli GC, Takemoto RM. Structure and Diversity of endoparasitic infracommunities and the trophic level of Pseudoplatystoma corruscans and Schizodon borelli (Osteichthyes) of the high Paraná River. Mem Inst Oswaldo Cruz 1996; 91(4): 441-448. http://dx.doi.org/10.1590/ S0074-02761996000400010. PMid:9070406.

Machado PM, Almeida SC, Pavanelli GC, Takemoto RM. Ecological aspects of endohelminths parasitizing Cichla monoculus Spix, 1831 (Perciformes: Cichlidae) in the Paraná River near Porto Rico, State of Paraná, Brazil. Comp Parasitol 2000; 6(2): 210-217.

Madi RR, Silva MSR. Contracaecum Railliet \& Henry, 1912 (Nematoda, Anisakidae): the parasitism related with the biology of three species of piscivorous fishes in the Jaguari reservoir, São Paulo State, Brazil. Rev Bras Zoociênc 2005; 7(1): 15-24.

Martins ML, Onaka EM, Fenerick J Jr. Larval Contracaecum sp. (Nematoda: Anisakidae) in Hoplias malabaricus and Hoplerythrinus unitaeniatus (Osteichthyes: Erythrinidae) of economic importance in occidental marshlands of Maranhão, Brazil. Vet Parasitol 2005; 127(1): 51-59. http://dx.doi.org/10.1016/j.vetpar.2004.09.026. PMid:15619375.

Martins ML, Santos RS, Takahashi HK, Marengoni NG, Fujimoto RY. Infection and susceptibility of three fish species from the Paraná River, Presidente Epitácio, State of São Paulo, Brazil, to Contracaecum sp. larvae (Nematoda: Anisakidae). Acta Sci Anim Sci 2003; 25(1): 73-78. http:// dx.doi.org/10.4025/actascianimsci.v25i1.2096.

Mattos DPBG, Lopes LMS, Verícimo MA, Alvares TS, São Clemente SC. Anisakidae larvae infection in five commercially important fish species from the State of Rio de Janeiro, Brazil. Rev Bras Med Vet 2014; 36(4): 375-379.
Mesquita RLB, Santos SMC, Ceccarelli OS, Luque JL. Metazoários endoparasitos de Salminus brasiliensis (Cuvier, 1816) (Characiformes: Characidae) do rio Mogi Guaçu, SP, Brasil. Rev Bras Zoociênc 2012; 14(1-3): 95-102.

Moravec F, Kohn A, Fernandes BMM. Nematode parasites of fishes of the Paraná River, Brazil. Part 2. Seuratoidea, Ascaridoidea, Habronematoidea and Acuarioidea. Folia Parasitol (Praha) 1993; 40(2): 115-134.

Moravec F, van Rensburg CJ, Van As LL. Larvae of Contracaecum sp. (Nematoda: Anisakidae) in the threatened freshwater fish Sandelia capensis (Anabantidae) in South Africa. Dis Aquat Organ 2016; 120(3): 251-254. http://dx.doi.org/10.3354/dao03033. PMid:27503922.

Moravec F. Experimental studies on the development of Contracaecum rudolphii (Nematoda: Anisakidae) in copepod and fish paratenic hosts. Folia Parasitol (Praha) 2009; 56(3): 185-193. http://dx.doi.org/10.14411/ fp.2009.023. PMid:19827362.

Moravec F. Nematodes of freshwater fishes of the Neotropical Region. Praha: Academy of Sciences of the Czech Republic; 1998

Moreira LHA, Takemoto RM, Yamada FH, Ceschini TL, Pavanelli GC. Ecological aspects of metazoan endoparasites of Metynnis lippincottianus (Cope, 1870) (Characidae) from Upper Paraná River floodplain, Brazil. Helminthologia 2009; 46(4): 214-219. http://dx.doi.org/10.2478/ s11687-009-0040-9.

Moreira ST, Ito KF, Takemoto RM, Pavanelli GC. Ecological aspects of the parasites of Iheringichthys labrosus (Lütken, 1874) (Siluriformes: Pimelodidae) in reservoirs of Paraná basin and upper Paraná floodplain, Brazil. Acta Sci Biol Sci 2005; 27(4): 317-322.

Neves LR, Pereira FB, Tavares-Dias M, Luque JL. Seasonal Influence on the parasite fauna of a wild population of Astronotus ocellatus (Perciformes: Cichlidae) from the Brazilian Amazon. J Parasitol 2013; 99(4): 718-721. http://dx.doi.org/10.1645/12-84.1. PMid:23421456.

Oliveira MSB, Corrêa LL, Prestes L, Neves LR, Brasiliense ARP, Ferreira DO, et al. Comparison of the endoparasite fauna of Hoplias malabaricus and Hoplerythrinus unitaeniatus (Erythrinidae), sympatric hosts in the eastern Amazon region (Brazil). Helminthologia 2018; 55(2): 157-165. http://dx.doi.org/10.2478/helm-2018-0003.

Oliveira MSB, Gonçalves RA, Ferreira DO, Pinheiro DA, Neves LR, Dias MKR, et al. Metazoan parasite communities of wild Leporinus friderici (Characiformes: Anostomidae) from Amazon River system in Brazil. Stud Neotrop Fauna Environ 2017; 52(2): 146-156. http://dx.doi.org/1 $0.1080 / 01650521.2017 .1312776$.

Oliveira MSB, Gonçalves RA, Neves LR, Tavares-Dias M. Parasitic endohelminths of Metynnis hypsauchen (Characidae) from Jari River basin, brazilian amazon. Neotrop Helminthol 2015; 9(2): 235-242.

Oliveira MSB, Gonçalves RA, Tavares-Dias M. Community of parasites in Triportheus curtus and Triportheus angulatus (Characidae) from a tributary of the Amazon River system (Brazil). Stud Neotrop Fauna Environ 2016; 51(1): 29-36. http://dx.doi.org/10.1080/01650521.2016.1150095.

Oliveira MSB, Tavares-Dias M. Communities of parasite metazoans in Piaractus brachypomus (Pisces, Serrasalmidae) in the lower Amazon River (Brazil). Rev Bras Parasitol Vet 2016; 25(2): 151-157. http://dx.doi. org/10.1590/S1984-29612016022. PMid:27334815.

Pantoja WMF, Silva LVF, Tavares-Dias M. Are similar the parasite communities structure of Trachelyopterus coriaceus and Trachelyopterus galeatus (Siluriformes: Auchenipteridae) in the Amazon basin? Rev Bras Parasitol Vet 2016; 25(1): 46-53. http://dx.doi.org/10.1590/S198429612016016 . PMid:27007248. 
Paraguassú AR, Alves DR, Luque JL. Metazoan parasites of the pearl cichlid, Geophagus brasiliensis (Quoy e Gaimard, 1824) (Osteichthyes: Cichlidae) from the Lajes Reservoir, Rio de Janeiro, Brazil. Rev Bras Parasitol Vet 2005; 14(1): 35-39. PMid:16153342.

Paraguassú AR, Luque JL, Alves DR. Community ecology of the metazoan parasites of red porgy, Pagrus pagrus (L., 1758) (Osteichthyes: Sparidae), from the coastal zone, state of Rio de Janeiro, Brazil. Acta Sci Biol Sci 2002; $24(2)$ : 461-467.

Paraguassú AR, Luque JL. Metazoários Parasitos de seis espécies de Peixes do Reservatório de Lajes, Estado do Rio de Janeiro, Brasil. Rev Bras Parasitol Vet 2007; 16(3): 121-128. http://dx.doi.org/10.1590/ S1984-29612007000300002. PMid:18078597.

Pavanelli GC, Eiras JE, Yamaguchi ME, Takemoto RM. Zoonoses humanas transmissiveis por peixes no Brasil. Maringá: UniCesumar; 2015.

Pavanelli GC, Karling LC, Takemoto RM, Ueda BH. Estado da arte dos parasitos de peixes de água doce do Brasil. In: Pavanelli GC, Takemoto RM, Eiras JC, orgs. Parasitologia de Peixes de água doce do Brasil. Maringá: Eduem; 2013. p. 11-16.

Pedro NHO, Pellegrini LS, Azevedo RK, Abdallah VD. Biodiversity of metazoan parasites in Acestrorhynchus lacustris (Lütken, 1875) (Characiformes: Acestrorhynchidae) from the Batalha River, São Paulo State, Brazil. Pan-Am J Aquat Sci 2016; 11(4): 336-344.

Rassier GL, Pesenti TC, Pereira J Jr, Silva DS, Wendt EW, Monteiro $\mathrm{CM}$, et al. Metazoan parasites of Geophagus brasiliensis (Perciformes: Cichlidae) in Patos lagoon, extreme south of Brazil. Rev Bras Parasitol Vet 2015; 24(4): 447-453. http://dx.doi.org/10.1590/S1984-29612015075. PMid:26648010.

Rego AA, Santos CP. Helmintofauna de cavalas, Scomber japonicus Houtt, do Rio de Janeiro. Mem Inst Oswaldo Cruz 1983; 78(4): 443-448. http:// dx.doi.org/10.1590/S0074-02761983000400008.

Rego AA, Vicente JJ, Santos CP, Wekid RM. Parasitas de anchovas Pomatomus saltatrix (L.) do Rio de Janeiro. Cienc Cult 1983; 35(9): 1329-1336.

Ribeiro TS, Ueda BH, Pavanelli GC, Takemoto RM. Endoparasite fauna of Brycon amazonicus and B. melanopterus (Characidae, Bryconinae) from Negro and Solimões rivers, Amazon, Brazil. Acta Amazon 2016; 46(1): 107-110. http://dx.doi.org/10.1590/1809-4392201502153.

Rodrigues LC, Santos ACG, Ferreira EM, Teófilo TS, Pereira DM, Costa FN. Parasitologic aspects of traíra (Hoplias malabaricus) from the São Bento city, MA. Arq Bras Med Vet Zootec 2017; 69(1): 264-268. http:// dx.doi.org/10.1590/1678-4162-8798.

Rodrigues MV, Pantoja JCF, Guimarães CDO, Benigno RNM, Palha MDC, Biondi GF. Prevalence for nematodes of hygiene-sanitary importance in fish from Colares Island and Vigia, Pará, Brazil. $R$ Bras Ci Vet 2015; 22(2): 124-128.

Saad CDR, Luque JL. Larvas de Anisakidae na musculatura do pargo, Pagrus pagrus, no Estado do Rio de Janeiro, Brasil. Rev Bras Parasitol Vet 2009;18(e1 Supl 1): 71-73. http://dx.doi.org/10.4322/rbpv.018e1014. PMid:20040196.

Saad CDR, Vieira FM, Luque JL. Larvae of Anisakidae Skrjabin \& Karokhin, 1945 (Nematoda, Ascaridoidea) in Lophius gastrophysus MirandaRibeiro, 1915 (Actinopterygii, Lophiidae) from the coastal zone of the state of Rio de Janeiro, Brazil. Neotrop Helminthol 2012; 6(2): 159-177.

Sabas CSS, Brasil-Sato MC. Helminth fauna parasitizing Pimelodus pohli (Actinopterygii: Pimelodidae) from the upper Sáo Francisco River, Brazil.
Rev Bras Parasitol Vet 2014; 23(3): 375-382. http://dx.doi.org/10.1590/ S1984-29612014067. PMid:25271459.

Salgado RL. Avaliação parasitológica do pescado fresco comercializado no sudeste do Pará. Pubvet 2011; 5(1): 992-998. http://dx.doi.org/10.22256/ pubvet.v5n1.993.

Santana HP, Anjos CS, Morais AM, Malta JCO. Nematode larvae with zoonotic importance found in peacock bass Cichla monoculus (Spix \& Agassiz, 1831) from floodplain lakes in Central Amazon. Rev Bras Zoociênc 2017; 18(2): 71-76.

Santos GM, Ferreira EJG, Zuanon JAS. Peixes comerciais de Manaus. Manaus: Ibama/AM; ProVárzea; 2006.

Santos-Clapp MD, Brasil-Sato MC. Comunidade parasitária de Cichla kelberi (Perciformes, Cichlidae) do Reservatório de Três Marias, Minas Gerais, Brasil. Rev Bras Parasitol Vet 2014; 23(3): 367-374. http://dx.doi. org/10.1590/S1984-29612014059. PMid:25271458.

São Clemente SC, Uchoa CMA, Serra Freire NM. Larvas de anisakideos em Pagrus pagrus (L.) e seu controle através de baixas temperaturas. Rev Bras Cienc Vet 1994; 1(1): 21-24. http://dx.doi.org/10.4322/rbcv.2015.006.

Saraiva A, Silva FA, Silva-Souza AT. Nematode parasites of the characid fish Brycon hilarii from the River Juba, Mato Grosso, Brazil. Helminthologia 2006; 43(3): 158-160. http://dx.doi.org/10.2478/s11687-006-0029-6.

Silva CM, São Clemente SC. Nematóides da família Anisakidae e cestóides da ordem Trypanorhyncha em filés de dourado (Coryphaena hippurus) e aricó (Lutjanus synagris) e sua importância na inspeção de pescado. Hig Aliment 2001; 15(80): 75-79.

Silva LO, Luque JL, Alves DR, Paraguassú AR. Community ecology of metazoan parasites of the atlantic cutlassfish Trichiurus lepturus Linnaeus (Osteichthyes: Trichiuridae) from the coastal zone of the state of Rio de Janeiro, Brazil. Rev Bras Zoociênc 2000; 2(2): 115-133.

Soares IA, Vieira FM, Luque JL. Parasite community of Pagrus pagrus (Sparidae) from Rio de Janeiro, Brazil: evidence of temporal stability. Rev Bras Parasitol Vet 2014; 23(2): 216-223. http://dx.doi.org/10.1590/ S1984-29612014047. PMid:25054501.

Takemoto RM, Amato JFR, Luque JL. Comparative analysis of the metazoan parasite communities of leatherjackets, Oligoplites palometa, $O$. saurus, and O. saliens (Osteichthyes: Carangidae) from Sepetiba Bay, Rio de Janeiro. Brazil. Rev Bras Biol 1996; 56(4): 639-650. PMid:9253203.

Takemoto RM, Lizama MAP. Helminth fauna of fishes from the Upper Paraná River floodplain, Brazil. Neotrop Helminthol 2010; 4(1): 5-8.

Takemoto RM, Pavanelli GC, Lizama MAP, Lacerda ACF, Yamada FH, Moreira LHA, et al. Diversity of parasites of fish from the Upper Paraná River floodplain, Brazil. Braz J Biol 2009;69(2 Suppl 2): 691705. PMid:19738975.

Tavares LER, Luque JL, Neto SLB. Ecologia da comunidade de metazoários parasitos do olho-de-cão Priacanthus arenatus (Cuvier, 1829) (Osteichthyes, Priacanthidae) do litoral do estado do Rio de Janeiro, Brasil. Rev Bras Zoociênc 2001; 3(1): 45-59.

Tavares LER, Luque JL. Community ecology of the metazoan parasites of white sea catfish, Netuma barba (Osteichthyes: Ariidae), from the coastal zone of the state of Rio de Janeiro, Brazil. Braz J Biol 2004; 64(1): 169-176. PMid:15195376.

Tavares-Dias M, Neves LR. Diversity of parasites in wild Astronotus ocellatus (Perciformes, Cichlidae), an ornamental and food fish in Brazil. An Acad Bras Cienc 2017;89(3 Suppl): 2305-2315. http://dx.doi. org/10.1590/0001-3765201720160700. PMid:29044314. 
Tavares-Dias M, Sousa TJSM, Neves LR. Parasitic infections in two benthopelagic fish from Amazon: the Arowana Osteoglossum bicirrhosum (Osteoglossidae) and Oscar Astronotus ocellatus (Cichlidae). Biosci J 2014; 30(2): 546-555.

Timi JT, Sardella NH, Navone GT. Parasitic nematodes of Engraulis anchoita Hubbs et Marini, 1935 (Pisces, Engraulidae) off the Argentine and Uruguayan coasts, South West Atlantic. Acta Parasitol 2001; 46(3): 186-193.

Travassos L, Artigas P, Pereira C. Fauna helmintologica dos peixes de água doce do Brasil. Arq Inst Biol (Sao Paulo) 1928; 1: 5-68.

Ubeira FM, Valinñas B, Lorenzo S, Iglesias R, Figueiras A, GarcíaVillaescusa R. Anisaquiosis y alergia: un estúdio seroepidemiológico en la comunidad autónoma Gallega. Gallega: Consellería de Sanidade e Servicios Sociais; 2000. (Documentos técnicos de salud pública).

Ventura AS, Ishikawa MM, Gabriel AMA, Silbiger HLN, Cavichiolo F, Takemoto RM. Histopathology from liver of tuvira (Gymnotus spp.) parasitized by larvae of nematodes. Cienc Rural 2016; 46(7): 1233-1239. http://dx.doi.org/10.1590/0103-8478cr20150881.

Ventura AS, Pádua SB, Ishikawa MM, Martins ML, Takemoto RM, Jerônimo GT. Endoparasites of Gymnotus sp. (Gymnotiformes: Gymnotidae) from commercial baitfish farming in Pantanal basin, Central Brazil. Bol Inst Pesca 2018; 44(3): e322. http://dx.doi.org/10.20950/1678-2305.2018.322.

Vicente JJ, Fernandes GL. Contribuição ao conhecimento dos helmintos de Bagre bagre (Linnaeus, 1766) Fowler, 1841 e de Macrodon ancylodon (Bloch, 1801) Jordan, Evermann \& Clark, 1930, no litoral da ilha de São Luis, Estado do Maranhão, Brasil. Bol Lab Hidrob Univ Maranhão 1978; 2(1): 91-96.

Vicente JJ, Pinto RM. Nematóides do Brasil. Nematóides de peixes. Atualização: 1985-1998. Rev Bras Zool 1999; 16(3): 561-610. http:// dx.doi.org/10.1590/S0101-81751999000300001.

Vicente JJ, Rodrigues HO, Gomes DC. Nematóides do Brasil. 1 1a parte: nematóides de peixes. Atas Soc Biol Rio de 1985; 25(1): 1-79.

Vicentin W, Vieira KRI, Costa FES, Takemoto RM, Tavares LER, Paiva F. Metazoan endoparasites of Serrasalmus marginatus (Characiformes: Serrasalminae) in the Negro River, Pantanal, Brazil. Rev Bras Parasitol Vet
2011; 20(1): 61-63. http://dx.doi.org/10.1590/S1984-29612011000100012. PMid:21439234.

Vicentin W, Vieira KRI, Tavares LER, Costa FES, Takemoto RM, Paiva F. Metazoan endoparasites of Pygocentrus nattereri (Characiformes: Serrasalminae) in the Negro River, Pantanal, Brazil. Rev Bras Parasitol Vet 2013; 22(3): 331-338. http://dx.doi.org/10.1590/S1984-29612013000300003. PMid:24142162.

Vieira DHMD, Caramello LE, Abadallah VD, Silva RJ, Azevedo RK. Community ecology of metazoan parasites of the sairú Cyphocharax nagelii from the Peixe River. Rev Bras Parasitol Vet 2013; 22(4): 611-615. http:// dx.doi.org/10.1590/S1984-29612013000400027. PMid:24473892.

Vieira-Menezes FG, Costa DPC, Brasil-Sato MC. Nematodes of Astyanax fasciatus (Actinopterygii: Characidae) and their parasitic indices in the São Francisco river, Brazil. Rev Bras Parasitol Vet 2017; 26(1): 10-16. http:// dx.doi.org/10.1590/s1984-29612016074. PMid:28177039.

Weiblen AM, Brandão DA. Levantamento parasitológico em Hoplias malabaricus, Bloch, (1749) (traíra) de águas da região de Santa Maria - RS. Cienc Rural 1992; 22(2): 203-208. http://dx.doi.org/10.1590/ S0103-84781992000200014.

Yamada FH, Bongiovani MF, Yamada POF, Silva RJ. Parasite infracommunities of Leporinus friderici: A comparison of three tributaries of the Jurumirim Reservoir in southeastern Brazil. An Acad Bras Cienc 2017; 89(2): 953-963. http://dx.doi.org/10.1590/0001-3765201720160554. PMid:28489196.

Yamada FH, Moreira LHA, Ceschini TL, Lizama MAP, Takemoto RM, Pavanelli GC. Parasitism associated with length and gonadal maturity stage of the freshwater fish Metynnis lippincottianus (Characidae). Neotrop Helminthol 2012; 6(2): 247-253.

Yamada FH, Takemoto RM. Metazoan parasite fauna of two peacock-bass cichlid fish in Brazil. Check List 2013; 9(6): 1371-1377. http://dx.doi. org/10.15560/9.6.1371.

Zago AC, Franceschini L, Zocoller-Seno MC, Veríssimo-Silveira R, Maia AAD, Ikefuti CV, et al. The helminth community of Geophagus proximus (Perciformes: Cichlidae) from a tributary of the Paraná River, Ilha Solteira Reservoir, São Paulo State, Brazil. J Helminthol 2013; 87(2): 203-211. http://dx.doi.org/10.1017/S0022149X12000223. PMid:22716964. 